\title{
Goursat Problem for a Microdifferential Operator of Fuchsian Type and Its Application
}

\author{
By
}

Susumu YAMAZAKI*

\begin{abstract}
We consider a matrix of microdifferential operators of Fuchsian type with respect to several variables and obtain the Cauchy-Kovalevskaja type theorem for the Goursat problem. Moreover, we prove the existence theorem in the framework of microfunctions under some assumptions for initial values and the operator.
\end{abstract}

\section{$\S 0 . \quad$ Introduction}

The Goursat problem in the holomorphic (or the real analytic) category is treated by several authors and studied in depth. Moreover, C. Wagschal [W] extended the problem to the case of a system of integro-differential operators and obtained the Cauchy-Kovalevskaja type (namely, the unique solvability) theorem. However, it seems that the study of the Goursat problem is not so satisfactory from the microlocal point of view. Therefore in this paper, we treat a microdifferential operator of Fuchsian type with respect to several variables and consider the Goursat problem in the framework of holomorphic (or micro-) functions.

The notion of Fuchsian type (with respect to one variable) was introduced by $\mathrm{M}$. S. Baouendi and C. Goulaouic [Ba-G] for a partial differential operator. This includes non characteristic type as a special case, and the Cauchy-Kovalevskaja type theorem was proved in [Ba-G]. Seeing this result, N. S. Madi [M] generalized Fuchsian type to several variable case by the name of "operateurs de Goursat holomorphes à plusieurs variables Fuchsiennes" and obtained the Cauchy-Kovalevskaja type theorem for the Goursat problem

Communicated by T. Kawai, August 23, 1996. Revised November 15, 1996 and January 23, 1997.

1991 Mathematics Subject Classification: Primary 35S10; Secondary 32A45, 35A27.

*Graduate School of Mathematical Sciences, The University of Tokyo, 8-1 Komaba 3-chome, Meguro-ku, Tokyo 153, Japan. 
in the framework of holomorphic functions. We remark that Y. LaurentT. Monterio Fernandes [La-MF] and Z. Szmydt and B. Ziemian [Sz-Zi] gave different definitions of Fuchsian type with respect to several variables respectively. On the other hand, after Baouendi-Goulaouic [Ba-G], many mathematicians have obtained almost sufficient results in Fuchsian type with respect to one variable. For example, $\mathbb{H}$. Tahara $[\mathrm{Ta}]$ treated a Fuchsian system in the sense of Volevič and proved the Cauchy-Kovalevskaja type theorem in the complex domain. Further, as an application he obtained the existence and uniqueness theorem on an initial value problem for a Fuchsian hyperbolic system in the framework of hyperfunctions. Moreover, he proved the existence theorem on a homogeneous initial value problem for a Fuchsian microhyperbolic system of microdifferential operators in the framework of microfunctions. On the other hand, T. Oaku proved the existence theorem on an inhomogeneous initial value problem for a Fuchsian hyperbolic microdifferential operator in [O2] (cf. [O1]) and the uniqueness theorem under the $F$-mildness condition (but without the hyperbolicity assumption) in [04] in the framework of microfunctions (cf. [O3]).

In this paper, we define a matrix of microdifferential operators of Fuchsian type with respect to several variables as a natural generalization of one variable case due to Tahara [Ta] or non-microlocal case due to Madi [M]. Moreover, we prove the Cauchy-Kovalevskaja type theorem for the Goursat problem in the space of holomorphic functions under the action of microdifferential operators due to J. M. Bony and P. Schapira [Bo-Sc]. As an application we solve the Goursat problem in the framework of micro-(or hyper-) functions; we prove the existence theorem for sufficiently "regular" initial data under suitable assumptions. Note that our solvability conditions are weaker than that of M. Kashiwara and P. Schapira [K-Sc1]. We hope our results will serve as a starting point for studying the Goursat problem for general systems in microlocal analysis.

We will show the plan of this paper.

In Section 1, we announce the main theorem on the Goursat problem in the complex domain after some preparations.

In Section 2, we reduce the problem to a simple case.

In Section 3, we give the proof of the main theorem.

Section 4 is devoted to applications to microlocal solvability for the Goursat problem under suitable assumptions. 


\section{Acknowledgments}

I would like to express my sincere gratitude to Professor Kiyoomi Kataoka for helpful discussions and suggestions. I would like to thank Professor Hikosaburo Komatsu for his encouragement, Professor Yasunori Okada and Professor Shinya Moritoh for fruitful discussions. Finally, I would like to thank the referee for carefully reading the manuscript and valuable comments.

\section{$\S 1$. Statement of Main Theorem}

In this paper, we use the following notation: $\mathbb{N}$ denotes the set of natural numbers (not containing 0 ) and $\mathbb{N}_{0}:=\mathbb{N} \cup\{0\}$. For a subset $D$ of some topological space, $[D]$ denotes the closure. For natural numbers $M, N \in N$, and a linear space $L$ we denote by $\operatorname{Mat}(M, N ; L)$ the space of matrices of size $M \times N$ whose components are in $L$. Further set

$$
\left\{\begin{aligned}
\operatorname{Mat}(N ; L) & :=\operatorname{Mat}(M, N ; L), \\
L^{N} & :=\operatorname{Mat}(1, N ; L), \\
L^{\oplus N} & :=\operatorname{Mat}(M, 1 ; L) .
\end{aligned}\right.
$$

In addition, if $L$ has a norm \|\| , for $P=\left(P^{(\mu, v)}\right)_{\mu, v=1}^{N} \in \operatorname{Mat}(M, N ; L)$ we set $\|P\|:=\max \left\{\left\|P^{(\mu, v)}\right\| ; 1 \leqslant \mu \leqslant M, 1 \leqslant v \leqslant N\right\}$. For natural numbers $d, n \in \mathbb{N}$, we use coordinates $\tau=\left(\tau_{1}, \ldots, \tau_{d}\right) \in C^{d}$ and $z=\left(z_{1}, \ldots, z_{n}\right) \in C^{n}$. Moreover for multi-indices $\gamma=\left(\gamma_{1}, \ldots, \gamma_{n}\right)$ and $\alpha=\left(\alpha_{1}, \ldots, \alpha_{d}\right)$, we set

$$
\left\{\begin{aligned}
\partial_{z}^{\gamma}:=\partial_{z_{1}}{ }^{\gamma_{1}} \cdots \partial_{z_{n}}^{\gamma_{n},}, & \partial_{\tau}^{\alpha}:=\partial_{\tau_{1}}^{\alpha_{1}} \cdots \partial_{\tau_{d}}^{\alpha_{d}}, \\
z^{\gamma}:=z_{1}^{\gamma_{1}} \cdots z_{n}^{\gamma_{n},} & \tau^{\alpha}:=\tau_{1}^{\alpha_{1}} \cdots \tau_{d}^{\alpha_{d}}, \\
\gamma !:=\gamma_{1} ! \cdots \gamma_{n} !, & \alpha !:=\alpha_{1} ! \cdots \alpha_{d} !, \\
|\gamma|:=\sum_{j=1}^{n} \gamma_{j}, & |\alpha|:=\sum_{j=1}^{d} \alpha_{j},
\end{aligned}\right.
$$

as usual. For vectors $R=\left(R_{1}, \ldots, R_{d}\right)$ and $R^{\prime}=\left(R_{1}^{\prime}, \ldots, R_{d}^{\prime}\right) \in \boldsymbol{R}^{d}$, we define an order relation as follows:

$$
\begin{aligned}
& R^{\prime} \leqslant R_{\text {def. }}^{R \Leftrightarrow R_{j}^{\prime} \leqslant R_{j}} \quad \text { for all } j, \\
& R^{\prime}<R \Leftrightarrow R^{\prime} \leqslant R \text { and } R^{\prime} \neq R, \\
& \text { def. }
\end{aligned}
$$




$$
R^{\prime} \prec R \Leftrightarrow \underset{\text { def. }}{R_{j}^{\prime}<R_{j} \quad \text { for all } j .}
$$

For a vector $r=\left(r_{1}, \ldots, r_{d}\right) \in \mathbb{R}^{d}$, we set $[r]_{+}:=\left(\left[r_{1}\right]_{+}, \ldots,\left[r_{d}\right]_{+}\right)$, where $\left[r_{j}\right]_{+}=\max \left\{r_{j}, 0\right\}$. We fix $m^{(v)}=\left(m_{1}^{(v)}, \ldots, m_{d}^{(v)}\right)$ and $k^{(v)}=\left(k_{1}^{(v)}, \ldots, k_{d}^{(v)}\right) \in N_{0}{ }^{d}$ with $m^{(v)} \geqslant k^{(v)}(1 \leqslant v \leqslant N)$ and set $m=\left(m^{(1)}, \ldots, m^{(N)}\right)$ and $k=\left(k^{(1)}, \ldots, k^{(N)}\right) \in\left(N_{0}{ }^{d}\right)^{N}$. For any $N$-tuple of (generalized) functions $f(z, \tau)={ }^{t}\left(f_{1}(z, \tau), \ldots, f_{N}(z, \tau)\right)$, we mean by $f=O\left(\tau^{m-k}\right)$

$$
\left.\partial_{\tau_{l}}^{j} f_{v}\right|_{\tau_{l}=0}=0 \quad\left(1 \leqslant v \leqslant N, 1 \leqslant i \leqslant d, 0 \leqslant j \leqslant m_{i}^{(v)}-k_{i}^{(v)}-1\right) .
$$

Set $\mathbb{1}_{d}:=(1, \ldots, 1) \in \mathbb{N}^{d}$. For a vector $R=\left(R_{1}, \ldots, R_{d}\right) \in \boldsymbol{R}^{d}$ with $0 \prec R$, we set

$$
B(R):=\left\{\tau \in C^{d} ;\left|\tau_{j}\right|<R_{j}(1 \leqslant j \leqslant d)\right\} .
$$

Let $V \subset C^{n}$ be a relatively compact open neighborhood of the origin and $h_{0}$ a positive number. We set

$$
U:=\left\{(z ; \zeta) \in T^{*} C^{n} ; z \in V, \zeta_{1}=1,\left|\zeta_{j}\right|<h_{0}(2 \leqslant j \leqslant n)\right\}
$$

We denote the sheaf of rings of microdifferential operators of finite order (resp. of order at most $v$ ) by $\mathscr{E}($ resp. $\mathscr{E}(v))$ as usual.

1.1. Definition. Let $\mathrm{P}\left(\mathrm{z}, \tau ; \partial_{z}, \partial_{\tau}\right)=\left(P^{(\mu, v)}\left(z, \tau ; \partial_{z}, \partial_{\tau}\right)\right)_{\mu, v=1}^{N}$ be a matrix in $\operatorname{Mat}\left(N ; \Gamma\left([U \times B(R)] ; \mathscr{E}_{C^{n+d}}\right)\right)$; that is, each $P^{(\mu, v)}$ is a microdifferential operator of finite order defined in some neighborhood of $[U \times B(R)]$. Then, $P$ is said to be of Fuchsian type with weight $(k, m)$ (with respect to $\tau$-variables) if it has the following form:

$$
P^{(\mu, v)}\left(z, \tau ; \partial_{z}, \partial_{\tau}\right)=\sum_{0 \leqslant \alpha \leqslant m^{(v)}} P_{\alpha}^{(\mu, v)}\left(z, \tau ; \partial_{z}\right) \partial_{\tau}^{\alpha},
$$

where each $P_{\alpha}^{(\mu, v)}$ is a microdifferential operator with holomorphic parameters $\tau$ and satisfies the following:

1. The order ord $P_{\alpha}^{(\mu, v)}$ of $P_{\alpha}^{(\mu, v)}$ is at most $\left|m^{(v)}\right|-|\alpha|$;

2. There exist $P_{\alpha}^{1,(\mu, v)}\left(z, \tau ; \partial_{z}\right)$ and $P_{\alpha}^{2 .(\mu, v)}\left(z, \tau ; \partial_{z}\right)\left(0 \leqslant \alpha \leqslant m^{(v)}\right)$ such that ord $P_{\alpha}^{1,(\mu, v)} \leqslant 0$ and

$$
\begin{aligned}
P_{\alpha}^{(\mu, v)}\left(z, \tau ; \partial_{z}\right)= & \tau^{\left[\alpha-m^{(v)}+k^{(v)}\right]+} P_{\alpha}^{1,(\mu, v)}\left(z, \tau ; \partial_{z}\right) \\
& +\tau^{\left[\alpha-m^{(v)}+k^{(v)}+\mathbf{1}_{d}\right]+} P_{\alpha}^{2 .(\mu, v)}\left(z, \tau ; \partial_{z}\right) .
\end{aligned}
$$


1.2. Remark. (1) The Fuchsian property above is invariant under any coordinate change of $z$-variables, or more generally an arbitrary quantized contact transformation for $(z ; \zeta)$-variables.

(2) The Fuchsian type defined in Definition 1.1 is a natural generalization of differential operators of Fuchsian type introduced by Madi [M]; that is, if $P$ is a differential operator of Fuchsian type in the sense of Definition 1.1, then $P$ is of Fuchsian type in the sense of Madi. Further if $d=N=1$, a microdifferential operator of Fuchsian type is nothing but of Fuchsian type defined by Tahara (see $[\mathrm{Ta}])$.

Let $T^{(v)}=\left(T_{1}^{(v)}, \ldots, T_{d}^{(v)}\right)(1 \leqslant v \leqslant N)$ be indeterminates and set

$$
\tilde{T}:=\left(T^{(1)}, \ldots, T^{(N)}\right) .
$$

If $P$ is of Fuchsian type with weight $(k, m)$, we define the indicial polynomial of $P$ by

$$
\mathscr{I}_{P}(z ; \zeta ; \tilde{T}):=\operatorname{det}\left(\sum_{m^{(v)}-k^{(v)} \leqslant \alpha \leqslant m^{(v)}} \sigma_{0}\left(P_{\alpha}^{1 .(\mu, v)}\right)(z, 0 ; \zeta) \mathscr{I}_{\alpha}\left(T^{(v)}\right)\right)
$$

where $\mathscr{I}_{\alpha}\left(T^{(v)}\right):=\prod_{j=1}^{d} \mathscr{I}_{\alpha_{j}}\left(T_{j}^{(v)}\right)$ with

$$
\mathscr{I}_{\alpha_{j}}\left(T_{j}^{(v)}\right):=\left\{\begin{array}{cc}
T_{j}^{(v)}\left(T_{j}^{(v)}-1\right) \cdots\left(T_{j}^{(v)}-\alpha_{j}+1\right) & \left(\alpha_{j} \geqslant 1\right), \\
1 & \left(\alpha_{j}=0\right) .
\end{array}\right.
$$

Let $A\left(z, \tau ; \partial_{z}\right)$ be a microdifferential operator of finite order with holomorphic parameters $\tau$ defined in a neighborhood of $[U \times B(R)]$. Let $c \in C$ and set $\Sigma:=\left\{z \in C^{n} ; z_{1}=c\right\}$. Let $\Omega \subset V$ be an open convex set and assume that $\Omega$ is $h_{0}-\Sigma$-flat in the sense of Bony-Schapira; that is, if $z \in \Omega, w \in \Sigma$ and $h_{0}\left|z_{j}-w_{j}\right| \leqslant\left|z_{1}-w_{1}\right|(2 \leqslant j \leqslant n)$, then it follows that $w \in \Omega \cap \Sigma$. For example, we easily see that for any $z_{0} \in \Sigma$ the set $\left\{w \in C^{n} ;\left|w_{1}-z_{1}\right| / h+\left|w_{j}-z_{j}\right|<r(2 \leqslant j \leqslant n)\right\}$ is $h$ - $\Sigma$-flat. Let $f(z, \tau)$ be a holomorphic function defined on $\Omega \times B(R)$. If $p \in N$, there exists a unique holomorphic function $g(z, \tau)$ on $\Omega \times B(R)$ such that

$$
\left\{\begin{array}{l}
\partial_{z_{1}}^{p} g(z, \tau)=f(z, \tau) \\
\left.\partial_{z_{1}}^{j} g\right|_{z_{1}=c}=0 \quad(0 \leqslant j \leqslant p-1) .
\end{array}\right.
$$


Then, we define $\left(\partial_{z_{1}}{ }^{-p}\right)_{\Sigma} f(z, \tau):=g(z, \tau)$; that is,

$$
\left(\partial_{z_{1}}{ }^{-p}\right)_{\Sigma} f(z, \tau):=\int_{c}^{z_{1}} \frac{\left(z_{1}-w_{1}\right)^{p-1}}{(p-1) !} f\left(w_{1}, z^{\prime}\right) d w_{1},
$$

where $z^{\prime}:=\left(z_{2}, \cdots, z_{n}\right)$. We write

$$
A\left(z, \tau ; \partial_{z}\right)=\sum_{\substack{\gamma_{1} \in \mathbb{Z} \\ \gamma_{2}, \cdots, \gamma_{n} \in N_{0}}} A_{\gamma}(z, \tau) \partial_{z}{ }^{\gamma}
$$

formally. Then, applying the argument in the proof of Theorème 2.3.3 of Bony-Schapira [Bo-Sc] regarding $\tau$ as holomorphic parameters, we find that

$$
A_{\Sigma} f(z, \tau):=\sum_{\gamma \in \mathbb{N}_{0} d^{d}} A_{\gamma}(z, \tau) \partial_{z}^{\gamma} f(z, \tau)+\sum_{\substack{\gamma_{1}<0 \\ \gamma_{2}, \cdots, \gamma_{n} \in N_{0}}} A_{\gamma}(z, \tau)\left(\partial_{z_{1}}{ }^{\gamma_{1}}\right)_{\Sigma} \partial_{z^{\prime}}{ }^{\gamma^{\prime}} f(z, \tau)
$$

is holomorphic on $\Omega \times B(R)$. Let $s$ be a parameter with $0<s<1$. We fix a point $z_{0} \in \Omega \cap \Sigma$ and set

$$
\Omega_{s}:=\left\{s\left(z-z_{0}\right)+z_{0} \in \mathbb{C}^{n} ; z \in \Omega\right\}
$$

We fix an arbitrary positive number $c_{0}$ and set

$$
X_{s}:=\left\{f(z) \in \mathcal{O}\left(\Omega_{s}\right) ;\|f\|_{s}:=\sup \left\{|f(z)| d_{s}^{\prime}(z)^{c_{0}} ; z \in \Omega_{s}\right\}<\infty\right\},
$$

where $\mathcal{O}\left(\Omega_{s}\right)$ denotes the space of holomorphic functions defined on $\Omega_{s}$ and

$$
d_{s}^{\prime}(z):=\inf \left\{\max _{2 \leqslant j \leqslant n}\left|z_{j}-w_{j}\right| ; w \in \mathbb{C}^{n} \backslash \Omega_{s}, \quad z_{1}=w_{1}\right\}
$$

Note that $X_{s}$ is a Banach space under the norm $\|f\|_{s}$ and that $X_{s^{\prime}} \subset X_{s}$ and $\|f\|_{s} \leqslant\|f\|_{s^{\prime}}$ for any $0<s<s^{\prime}<1$ and $f \in X_{s^{\prime}}$. Moreover, by Proposition 2.4.3 of Bony-Schapira [Bo-Sc], we see that if the operator $A\left(z, \tau ; \partial_{z}\right)$ above is of order zero then $A_{\Sigma}$ operates on $X_{s}$ as a bounded linear operator; that is, there exists a constant $C$ such that for any $f \in X_{s}$,

$$
\sup \left\{\left\|\left(A_{\Sigma} f\right)(\cdot, \tau)\right\|_{s} ; \tau \in B(R)\right\} \leqslant C\|f\|_{s} .
$$


Consider the following condition:

[A-11. There exist a positive constant $C>0$ and a neighborhood $W$ of $[U]$ such that for any $(z ; \zeta) \in[W]$ and $\beta \in N_{0}{ }^{d}$

$$
\left|\mathscr{I}_{P}\left(z ; \zeta ; \beta+m^{(1)}-k^{(1)}, \ldots, \beta+m^{(N)}-k^{(N)}\right)\right| \geqslant C \prod_{v=1}^{N}\left(\beta+\mathbb{1}_{d}\right)^{m^{(v)}} .
$$

Note that if $N=1$, then [A-1] is a natural generalization of Madi's condition which is similar to the "Fuchsian ellipticity condition" due to Szmydt-Ziemian [Sz-Zi].

1.3. Theorem. Let $P$ be a matrix of microdifferential operators defined in a neighborhood of $[U \times B(R)]$. Assume that $P$ is of Fuchsian type with weight $(k, m)$ and satisfies $[\mathrm{A}-1]$. Then, there exist constants $r_{0}>0$ and $\stackrel{\circ}{R}$ with $0<\stackrel{R}{R} \leqslant R$ such that the following hold:

Take arbitrary $h$ and $r$ with $0<h<h_{0}$ and $0<r<r_{0}$ respectively. Let $\Omega$ be any $h$ - $\Sigma$-flat open convex subset of $V$ with dia $\Omega \leqslant r$, where dia denotes the diameter. Then, there exists a constant $\delta$ such that for any $\tilde{R}$ with $0<\tilde{R} \leqslant R$ it follows that for any holomorphic functions $f(z, \tau)={ }^{t}\left(f_{1}(z, \tau), \ldots, f_{N}(z, \tau)\right)$ and $g(z, \tau)={ }^{t}\left(g_{1}(z, \tau), \ldots, g_{N}(z, \tau)\right)$ on $\Omega \times B(\widetilde{R})$, there exists a unique holomorphic solution $u(z, \tau)={ }^{t}\left(u_{1}(z, \tau), \ldots, u_{N}(z, \tau)\right)$ of the Goursat problem

$$
\left\{\begin{aligned}
P_{\Sigma} u & =f \\
u-g & =O\left(\tau^{m-k}\right)
\end{aligned}\right.
$$

and each $u_{v}(z, \tau)(1 \leqslant v \leqslant N)$ is holomorphic on

$$
\bigcup_{0<s<1}\left(\Omega_{s} \times\left\{\tau \in B(\tilde{R}) ; \prod_{j=1}^{d}\left|\tau_{j}\right|<\delta(1-s)^{|m|}\right\}\right),
$$

where $|m|:=\sum_{v=1}^{N} \sum_{j=1}^{d} m_{j}^{(v)}$.

1.4. Remark. (1) Assume that $P$ is a differential operator. Then Theorem 1.3 is (essentially) obtained by Madi [M] (cf. [La-MF]).

(2) By definition (G.P.) is written as 


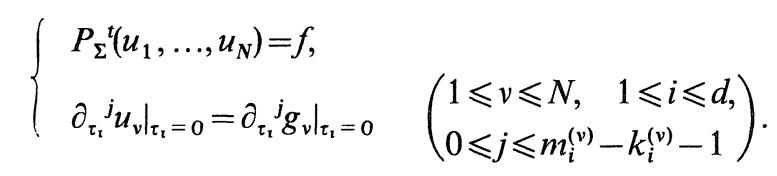

Let us set

$$
\Omega \times B(\widetilde{R})_{i}:=\Omega \times B(\widetilde{R}) \cap\left\{\tau_{i}=0\right\} \quad(1 \leqslant i \leqslant d)
$$

and regard each $\Omega \times B(\widetilde{R})_{i}$ as an open subset of $C^{n+d-1}$. Similarly we identify $\Omega \times B(\tilde{R}) \cap\left\{\tau_{1}=\cdots=\tau_{d}=0\right\}$ with $\Omega$. Let $g_{v, i j}(1 \leqslant v \leqslant N, \quad 1 \leqslant i \leqslant d$, $0 \leqslant j \leqslant m_{i}^{(v)}-k_{i}^{(v)}-1$ ) be holomorphic functions on $\Omega \times B(\widetilde{R})_{i}$ with compatibility conditions

(C.C.) $\left.\quad \partial_{\tau_{v^{\prime}}}{ }^{j^{\prime}} g_{v, i j}\right|_{\tau_{\tau^{\prime}}=0}=\left.\partial_{\tau_{1}}{ }^{j} g_{v, i^{\prime} j^{\prime}}\right|_{\tau_{l}=0} \quad\left(\begin{array}{l}1 \leqslant v \leqslant N, \quad 1 \leqslant i, i^{\prime} \leqslant d, \\ 0 \leqslant j \leqslant m_{i}^{(v)}-k_{i}^{(v)}-1, \\ 0 \leqslant j^{\prime} \leqslant m_{i^{\prime}}^{(v)}-k_{i^{\prime}}^{(v)}-1\end{array}\right)$.

Then we see that the Goursat problem (G.P.) is equivalent to the following problem:

$$
\left\{\begin{aligned}
P_{\Sigma}{ }^{t}\left(u_{1}, \ldots, u_{N}\right) & =f \\
\left.\partial_{\tau_{1}}{ }^{j} u_{v}\right|_{\tau_{i}=0} & =g_{v, i j} \quad\left(1 \leqslant v \leqslant N, \quad 1 \leqslant i \leqslant d, \quad 0 \leqslant j \leqslant m_{i}^{(v)}-k_{i}^{(v)}-1\right) .
\end{aligned}\right.
$$

Indeed, for $g(z, \tau)={ }^{t}\left(g_{1}(z, \tau), \cdots, g_{N}(z, \tau)\right)$ it is clear that the data

$$
\left\{g_{v, i j}=\left.\partial_{\tau_{1}}{ }^{j} g_{v}\right|_{\tau_{1}=0} \in \mathcal{O}\left(\Omega \times B(\tilde{R})_{i}\right) ; 1 \leqslant v \leqslant N, 1 \leqslant i \leqslant d, 0 \leqslant j \leqslant m_{i}^{(v)}-k_{i}^{(v)}-1\right\}
$$

satisfy (C.C.). Conversely, for any

$$
\left\{g_{v, i j}=\mathcal{O}\left(\Omega \times B(\tilde{R})_{i}\right) ; 1 \leqslant v \leqslant N, 1 \leqslant i \leqslant d, 0 \leqslant j \leqslant m_{i}^{(v)}-k_{i}^{(v)}-1\right\}
$$

which satisfy (C.C.), let us set

$$
g_{v}(z, \tau):=\sum_{\alpha \in N_{v}} \frac{g_{v, \alpha}(z)}{\alpha !} \tau^{\alpha}
$$

where

$$
N_{v}:=N_{0}^{d} \backslash\left\{\alpha \in N_{0}^{d} ; \alpha \geqslant m^{(v)}-k^{(v)}\right\}
$$

and 


$$
g_{v, \alpha}(z):=\left\{\begin{array}{cl}
\left.\partial_{\tau}^{\hat{\alpha}_{1}} g_{v, 1 \alpha_{1}}\right|_{\Omega} & \left(0 \leqslant \alpha_{1} \leqslant m_{1}^{(v)}-k_{1}^{(v)}-1\right), \\
\left.\partial_{\tau}^{\hat{\alpha}_{2}} g_{v, 2 \alpha_{2}}\right|_{\Omega} & \left(m_{1}^{(v)}-k_{1}^{(v)} \leqslant \alpha_{1}, 0 \leqslant \alpha_{2} \leqslant m_{2}^{(v)}-k_{2}^{(v)}-1\right), \\
\left.\partial_{\tau}^{\hat{\alpha}_{3}} g_{v, 3 \alpha_{3}}\right|_{\Omega} & \left(\begin{array}{l}
m_{1}^{(v)}-k_{1}^{(v)} \leqslant \alpha_{1}, m_{2}^{(v)}-k_{2}^{(v)} \leqslant \alpha_{2}, \\
0 \leqslant \alpha_{3} \leqslant m_{3}^{(v)}-k_{3}^{(v)}-1
\end{array}\right), \\
\vdots & \vdots \\
\left.\partial_{\tau}^{\hat{\alpha}_{d}} g_{v, d \alpha_{d}}\right|_{\Omega} & \left(\begin{array}{l}
m_{i}^{(v)}-k_{i}^{(v)} \leqslant \alpha_{i}(1 \leqslant i \leqslant d-1) \\
0 \leqslant \alpha_{d} \leqslant m_{d}^{(v)}-k_{d}^{(v)}-1
\end{array}\right),
\end{array}\right.
$$

with $\hat{\alpha}_{i}:=\left(\alpha_{1}, \ldots, \alpha_{i-1}, \stackrel{i}{0}, \alpha_{i+1}, \ldots, \alpha_{d}\right)$. Then we easily see that each $g_{v}(z, \tau)$ is holomorphic on $\Omega \times B(\tilde{R})$ and satisfies

$$
\left.\partial_{\tau_{i}}^{j} g_{v}\right|_{\tau_{\imath}=0}=g_{v, i j} \quad\left(1 \leqslant v \leqslant N, 1 \leqslant i \leqslant d, 0 \leqslant j \leqslant m_{i}^{(v)}-k_{i}^{(v)}-1\right) .
$$

Note that for example if $d=2$ or 3 , then each $g_{v}(z, \tau)$ is written explicitly as

$$
\begin{aligned}
g_{v}(z, \tau)= & \sum_{\alpha_{1}=0}^{m_{1}^{(v)}-k_{1}^{(v)}-1} \frac{g_{v, 1 \alpha_{1}}\left(z, \tau_{2}\right)}{\alpha_{1} !} \tau_{1}^{{ }^{\alpha}}+\sum_{\alpha_{2}=0}^{m_{2}^{(v)}-k_{2}^{(v)}-1} \frac{g_{v, 2 \alpha_{2}}\left(z, \tau_{1}\right)}{\alpha_{2} !} \tau_{2}{ }^{\alpha_{2}} \\
& -\sum_{0 \leqslant \alpha \leqslant m^{(v)}-k^{(v)-1_{2}}} \frac{\partial_{\tau_{1}}{ }^{\alpha_{1}} g_{v, 2 \alpha_{2}}(z, 0)}{\alpha !} \tau^{\alpha},
\end{aligned}
$$

or

$$
\begin{aligned}
& g_{v}(z, \tau)=\sum_{\alpha_{1}=0}^{m_{1}^{(v)}-k_{1}^{(v)}-1} \frac{g_{v, 1 \alpha_{1}}\left(z, \tau_{2}, \tau_{3}\right)}{\alpha_{1} !} \tau_{1}^{\alpha_{1}}+\sum_{\alpha_{2}=0}^{m_{2}^{(v)}-k_{2}^{(v)}-1} \frac{g_{v, 2 \alpha_{2}}\left(z, \tau_{1}, \tau_{3}\right)}{\alpha_{2} !} \tau_{2}{ }^{\alpha_{2}} \\
& +\sum_{\alpha_{3}=0}^{m_{3}^{(v)}-k_{3}^{(v)}-1} \frac{g_{v \cdot 3 \alpha_{3}}\left(z, \tau_{1}, \tau_{2}\right)}{\alpha_{3} !} \tau_{3}^{\alpha_{3}} \\
& -\sum_{\substack{0 \leqslant \alpha_{1} \leqslant m_{i}^{(v)}-k^{(v)}-1 \\
0 \leqslant \alpha_{2} \leqslant m_{2}^{(v)}-k_{2}^{(v)}-1}} \frac{\partial_{\tau_{1}}{ }^{\alpha_{1}} g_{v, 2 \alpha_{2}}\left(z, 0, \tau_{3}\right)}{\alpha_{1} ! \alpha_{2} !} \tau_{1}{ }^{\alpha_{1}} \tau_{2}{ }^{\alpha_{2}} \\
& -\sum_{\substack{0 \leqslant \alpha_{2} \leqslant m_{2}^{(v)}-k_{2}^{(v)}-1 \\
0 \leqslant \alpha_{3} \leqslant m_{3}^{(v)}-k_{3}^{(v)}-1}} \frac{\partial_{\tau_{2}}{ }^{\alpha_{2}} g_{v, 3 \alpha_{3}}\left(z, \tau_{1}, 0\right)}{\alpha_{2} ! \alpha_{3} !} \tau_{2}{ }^{\alpha_{2}} \tau_{3}{ }^{\alpha_{3}} \\
& -\sum_{\substack{0 \leqslant \alpha_{1} \leqslant m_{1}^{(v)}-k_{k}^{(v)}-1 \\
0 \leqslant \alpha_{3} \leqslant m_{3}^{(v)}-k_{3}^{(v)}-1}} \frac{\partial_{\tau_{3}}{ }^{\alpha_{3}} g_{v, 1 \alpha_{1}}\left(z, \tau_{2}, 0\right)}{\alpha_{3} ! \alpha_{1} !} \tau_{3}{ }^{\alpha_{3}} \tau_{1}{ }^{\alpha_{1}} \\
& +\sum_{0 \leqslant \alpha \leqslant m^{(v)}-k^{(v)}-1_{3}} \frac{\partial_{\tau_{1}}^{\alpha_{1}} \partial_{\tau_{2}}^{\alpha_{2}} g_{v .3 \alpha_{3}}(z, 0)}{\alpha !} \tau^{\alpha}
\end{aligned}
$$


respectively.

1.5. Example. (1) Let us set $m^{(v)}:=(2, \ldots, 2)$ and $k^{(v)}:=(1, \ldots, 1) \in \mathbb{N}_{0}{ }^{d}$ $(1 \leqslant v \leqslant N)$ and consider

$$
P\left(z, \tau ; \partial_{z}, \partial_{\tau}\right)=\prod_{j=1}^{d}\left(\tau_{j} \partial_{\tau_{j}}{ }^{2} I_{N}+A_{j}\left(z, \tau ; \partial_{z}\right) \partial_{\tau_{j}}+B_{j}\left(z, \tau ; \partial_{z}\right)\right)+C\left(z, \tau ; \partial_{z}\right) .
$$

Here

$$
\left\{\begin{aligned}
A_{j}\left(z, \tau ; \partial_{z}\right), & B_{j}\left(z, \tau ; \partial_{z}\right) \in \operatorname{Mat}(N ; \mathscr{E}(0)) \\
C\left(z, \tau ; \partial_{z}\right) & \in \operatorname{Mat}(N ; \mathscr{E}(2 d))
\end{aligned}\right.
$$

and $I_{N} \in \operatorname{Mat}(N ; \mathbb{R})$ denotes the identity matrix. Then, $P$ is of Fuchsian type with weight $\left(\left(k^{(1)}, \ldots, k^{(N)}\right),\left(m^{(1)}, \ldots, m^{(N)}\right)\right)$ and

$$
\mathscr{I}_{P}\left(z ; \zeta \beta+m^{(1)}-k^{(1)}, \ldots, \beta+m^{(N)}-k^{(N)}\right)=\prod_{j=1}^{d} \operatorname{det}\left(\left(\beta_{j}+1\right) \beta_{j}+\sigma_{0}\left(A_{j}\right)(z, 0 ; \zeta)\left(\beta_{j}+1\right)\right) .
$$

Hence if each eigenvalue of $\left(\sigma_{0}\left(A_{j}\right)(0,0 ; 1,0, \ldots, 0)\right)_{i, j=1}^{d}$ is not in $\{l \in \mathbb{Z} ; l \leqslant 0\}$, we see that $P$ satisfies the condition $[\mathrm{A}-1]$.

(2) Let us choose $N=d$ and set $m^{(v)}:=(0,0, \ldots, 0, \stackrel{\nu}{2}, 0, \ldots, 0)$ and $k^{(v)}:=(0,0$, $\ldots, 0, \stackrel{v}{1}, 0, \ldots, 0) \in \mathbb{N}_{0}{ }^{d}(1 \leqslant v \leqslant d)$. Consider

$$
\begin{aligned}
P\left(z, \tau ; \partial_{z}, \partial_{\tau}\right)= & \left(P^{(i, j)}\left(z, \tau ; \partial_{z}, \partial_{\tau}\right)\right)_{i, j=1}^{d} \\
= & \left(\tau_{j} \partial_{\tau_{j}}{ }^{2} \delta_{i j}+\left(A_{i j}\left(z, \tau ; \partial_{z}\right)+\tau^{\mathbb{1}_{d}} B_{i j}\left(z, \tau ; \partial_{z}\right)\right) \partial_{\tau_{j}}\right. \\
& \left.+C_{i j}\left(z, \tau ; \partial_{z}\right)+\tau^{\mathbb{1}_{d}} D_{i j}\left(z, \tau ; \partial_{z}\right)\right)_{i, j=1}^{d}
\end{aligned}
$$

where $A_{i j}\left(z, \tau ; \partial_{z}\right), B_{i j}\left(z, \tau ; \partial_{z}\right), C_{i j}\left(z, \tau ; \partial_{z}\right)$ and $D_{i j}\left(z, \tau ; \partial_{z}\right)$ are microdifferential operators with ord $A_{i j}$, ord $C_{i j} \leqslant 0$, ord $B_{i j} \leqslant 1$ and ord $D_{i j} \leqslant 2$ respectively. Then, $P$ is of Fuchsian type with weight $\left(\left(k^{(1)}, \ldots, k^{(N)}\right),\left(m^{(1)}, \ldots, m^{(N)}\right)\right)$ and $\mathscr{I}_{P}\left(z ; \zeta ; \beta+m^{(1)}-k^{(1)}, \ldots, \beta+m^{(N)}-k^{(N)}\right)=\operatorname{det}\left(\left(\beta_{j}+1\right) \beta_{j} \delta_{i j}+\sigma_{0}\left(A_{i j}\right)(z, 0 ; \zeta)\left(\beta_{j}+1\right)\right)$.

Hence if each eigenvalue of $\sigma_{0}\left(A_{i j}\right)(0,0 ; 1,0, \ldots, 0)$ is not in $\{l \in \mathbb{Z} ; l \leqslant 0\}$ and each $\left(\sigma_{0}\left(A_{i j}\right)\right)_{i, j=1}^{d}$ is an upper triangular matrix, we see that $\mathbb{P}$ satisfies the condition $[\mathrm{A}-1]$. 


\section{§2. Preliminaries}

In this section, we will make some reductions.

Considering

$$
\left\{\begin{aligned}
P_{\Sigma} u & =f-P_{\Sigma} g \\
u & =O\left(\tau^{m-k}\right)
\end{aligned}\right.
$$

we may assume that $g \equiv 0$. Further, if $P$ is of Fuchsian type with weight $(k, m)$ and $k \neq m$, then define $P_{1}$ by

$$
P_{1}:=P \cdot\left(\begin{array}{ccc}
\tau^{m_{1}-k_{1}} & & 0 \\
& \ddots & \\
0 & & \tau^{m_{N}-k_{N}}
\end{array}\right)
$$

Since a direct calculation shows that $P_{1}$ is of Fuchsian type with weight $(m, m)$ and $\mathscr{I}_{P_{1}}(z ; \zeta ; \beta, \ldots, \beta)=\mathscr{I}_{P}\left(z ; \zeta ; \beta+m^{(1)}-k^{(1)}, \ldots, \beta+m^{(N)}-k^{(N)}\right)$, we may assume that $k=m$. In this case, we can write $P$ as

$$
\begin{aligned}
P^{(\mu, v)}\left(z, \tau ; \partial_{z}, \partial_{\tau}\right)= & \sum_{0 \leqslant \alpha \leqslant m^{(v)}} P_{\alpha}^{1 .(\mu, v)}\left(z, \tau ; \partial_{z}\right) \tau^{\alpha} \partial_{\tau}{ }^{\alpha} \\
& +\sum_{0 \leqslant \alpha \leqslant m^{(v)}} P_{\alpha}^{2,(\mu, v)}\left(z, \tau ; \partial_{z}\right) \tau^{\alpha+1_{d}} \partial_{\tau}{ }^{\alpha}
\end{aligned}
$$

and for simplicity set

$$
\mathscr{I}_{P}(z ; \zeta ; \beta):=\mathscr{I}_{P}(z ; \zeta ; \beta, \ldots, \beta)
$$

Moreover, $P$ can be written as $P\left(z, \tau, \partial_{z}, \partial_{\tau}\right)=Q+Q^{\prime}+A$, where

$$
\begin{aligned}
Q^{(\mu, v)} & =\sum_{0 \leqslant \alpha \leqslant m^{(v)}} Q_{\alpha}^{(\mu, v)}\left(z ; \partial_{z}\right) \tau^{\alpha} \partial_{\tau}^{\alpha}, \\
Q^{(\mu, v)} & =\sum_{0 \leqslant \alpha \leqslant m^{(v)}} \sum_{i=1}^{d} \tau_{i} Q_{i, \alpha}^{(\mu, v)}\left(z, \tau ; \partial_{z}\right)\left(\partial_{\tau} \tau\right)^{\alpha}, \\
A^{(\mu, v)} & =\sum_{0 \leqslant \alpha \leqslant m^{(v)}} \tau^{1_{d}} A_{\alpha}^{(\mu, v)}\left(z, \tau ; \partial_{z}\right)\left(\partial_{\tau} \tau\right)^{\alpha} .
\end{aligned}
$$


Here $Q_{\alpha}^{(\mu, v)}, Q_{i, \alpha}^{\prime(\mu, v)}$ and $A_{\alpha}^{(\mu, v)}$ are microdifferential operators with ord $Q_{\alpha}^{(\mu, v)}$, ord $Q_{l, \alpha}^{\prime(\mu, v)} \leqslant 0$ and ord $A_{\alpha}^{(\mu, v)} \leqslant\left|m^{(v)}\right|-|\alpha|$, and $\left(\partial_{\tau} \tau\right)^{\alpha}$ denotes $\prod_{j=1}^{d}\left(\partial_{\tau_{j}} \tau_{j}\right)^{\alpha_{j}}$. Further, $Q$ satisfies [A-1]. Indeed, we have only to remark the following:

(1) We can write

$$
P_{\alpha}^{1 .(\mu, v)}\left(z, \tau ; \partial_{z}\right)=P_{\alpha}^{1 .(\mu, v)}\left(z, 0 ; \partial_{z}\right)+\sum_{i=1}^{d} \tau_{i} Q_{i, \alpha}^{\prime \prime(\mu, v)}\left(z, \tau ; \partial_{z}\right)
$$

with $\operatorname{ord} P_{\alpha}^{1,(\mu, v)}$, ord $Q_{i, \alpha}^{\prime \prime(\mu, v)} \leqslant 0$.

(2) Since ord $P_{m^{(v)}}^{2,(\mu, v)} \leqslant 0$, we can regard $\tau^{1_{d}} P_{m^{(v)}}^{2,(\mu, v)}\left(z, \tau ; \partial_{z}\right)$ as included in $\sum_{i=1}^{d} \tau_{i} Q_{i, \alpha}^{\prime \prime(\mu, \nu)}\left(z, \tau ; \partial_{z}\right)$

(3) We have

$$
\tau_{i} \tau_{j} \partial_{\tau_{\imath}} u= \begin{cases}\tau_{i} \partial_{\tau_{\imath}}\left(\tau_{j} u\right) & (i \neq j), \\ \left(\tau_{i} \partial_{\tau_{\imath}}-1\right)\left(\tau_{j} u\right) & (i=j)\end{cases}
$$

and

$$
\tau_{i}^{j} \partial_{\tau_{i}}^{j}=\left(\partial_{\tau_{\imath}} \tau_{i}-1\right) \cdots\left(\partial_{\tau_{i}} \tau_{i}-j\right)
$$

Let $\mathcal{O}\left(B(R) ; X_{s}\right)$ be the set of $X_{s}$-valued holomorphic functions defined on $B(R)$. Then, we easily see that

$$
\begin{aligned}
& \mathcal{O}\left(B(R) ; X_{s}\right)=\left\{f(z, \tau) \in \mathcal{O}\left(\Omega_{s} \times B(R)\right) ; 0<\forall R^{\prime} \prec R,\right. \\
&\left.\sup \left\{|f(z, \tau)| d_{s}^{\prime}(z)^{c_{0}} ; z \in \Omega_{s}, \tau \in B\left(R^{\prime}\right)\right\}<\infty\right\} .
\end{aligned}
$$

Therefore it follows that for any $0<s<1$

$$
\mathcal{O}(\Omega \times B(R)) \subset \mathcal{O}\left(B(R) ; X_{\mathrm{s}}\right) .
$$

Hence Theorem 1.3 is reduced to the following theorem:

2.1. Theorem. Let $P$ be a matrix of microdifferential operators of Fuchsian type with weight $(m, m)$. Assume that $P$ satisfies [A-1]. Then, there exist constants $r_{0}>0$ and $R$ with $0<\stackrel{\circ}{R} \leqslant R$ such that the following hold:

Take arbitrary $h$ and $r$ such that $0<h<h_{0}$ and $0<r<r_{0}$ respectively. Let $\Omega$ be any $h-\Sigma$-flat open convex subset of $V$ with dia $\Omega \leqslant r$. Then, there exists

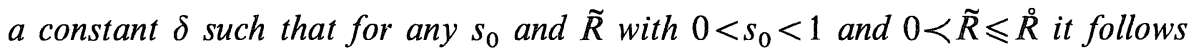
that for any $f(z, \tau)={ }^{t}\left(f_{1}(z, \tau), \ldots, f_{N}(z, \tau)\right) \in \mathcal{O}\left(B(\tilde{R}) ; X_{s_{0}}\right)^{\oplus N}$, there exists a unique 
holomorphic solution $u(z, \tau)={ }^{t}\left(u_{1}(z, \tau), \ldots, u_{N}(z, \tau)\right)$ of the Goursat problem (G.P.)

$$
P_{\Sigma} u=f
$$

Moreover, for any $s$ with $0<s<s_{0}$, each $u_{v}(z, \tau)(1 \leqslant v \leqslant N)$ is holomorphic on $\Omega_{s} \times B\left(R^{\prime}\right)$, where $R^{\prime}$ is an arbitraty vector which satisfies $0 \prec R^{\prime} \prec \tilde{R}$ and $\prod_{j=1}^{d} R_{j}^{\prime}<\delta\left(s_{0}-s\right)^{|m|}$.

In fact, if we prove Theorem 2.1 , we see that $u(z, \tau)$ is holomorphic on

$$
\begin{aligned}
& \bigcup_{\substack{0<s_{0}<1 \\
0<R^{\prime}<\tilde{R}}} \bigcup_{0<s<s_{0}}\left(\Omega_{s} \times\left\{\tau \in B\left(R^{\prime}\right) ; \prod_{j=1}^{d}\left|\tau_{j}\right|<\delta\left(s_{0}-s\right)^{|m|}\right\}\right) \\
& \quad=\bigcup_{0<s<1}\left(\Omega_{s} \times\left\{\tau \in B(\tilde{R}) ; \prod_{j=1}^{d}\left|\tau_{j}\right|<\delta(1-s)^{|m|}\right\}\right)
\end{aligned}
$$

by the uniqueness. Hence we obtain Theorem 1.3.

Let $u(T)=\sum_{\alpha \in N_{0} d} u_{\alpha} T^{\alpha}$ and $U(T)=\sum_{\alpha \in N_{0} d} U_{\alpha} T^{\alpha}$ be formal power series with indeterminaties $T=\left(T_{1}, \ldots, T_{d}\right)$, where $u_{\alpha} \in X_{s}$ and $U_{\alpha} \geqslant 0$. We say that $U(T)$ is a majorant of $u(T)$ if $\left\|u_{\alpha}\right\| \leqslant U_{\alpha}$ for any $\alpha$. In this case, we write $u(T) \ll U(T)$.

Define

$$
E\left(B(R) ; X_{s}\right):=\left\{u=\sum_{\alpha \in N_{0}{ }^{d}} u_{\alpha} T^{\alpha} \in X_{s}[[T]] ; \exists C>0, u(T) \ll C \Phi_{R}(T)\right\},
$$

where $\Phi_{R}(T)$ denotes $\prod_{j=1}^{d} \frac{R_{j}}{R_{j}-T_{j}}$. Then, we see the following (see Wagschal [W] for details):

1. $E\left(B(R) ; X_{s}\right)$ becomes a Banach space under the norm

$$
\|u\|_{R, s}:=\inf \left\{C ; u(T) \ll C \Phi_{R}(T)\right\} ;
$$

2. if $0<R \leqslant R^{\prime}$ and $0<s \leqslant s^{\prime}<1$, then it follows that $E\left(B\left(R^{\prime}\right) ; X_{s^{\prime}}\right)$ $\subset E\left(B(R) ; X_{s}\right)$ and

$$
\|u\|_{R, s} \leqslant\|u\|_{R^{\prime}, s^{\prime}}
$$

3. as a set 


$$
\bigcap_{0 \prec R^{\prime} \prec R} E\left(B\left(R^{\prime}\right) ; X_{s}\right)=\mathcal{O}\left(B(R) ; X_{s}\right)
$$

Hereafter, we also use the notation

$$
u(z, \tau)=\sum_{\alpha \in N_{0}{ }^{d}} u_{\alpha}(z) \tau^{\alpha}
$$

for

$$
u(z, T)=\sum_{\alpha \in N_{0} d} u_{\alpha}(z) T^{\alpha} \in E\left(B(R) ; X_{s}\right)
$$

We prove some results about Bony-Schapira's action on $E\left(B(R) ; X_{s}\right)$ :

2.2. Proposition. Let $A\left(z, \tau, ; \partial_{z}\right)$ be a microdifferential operator of order at most $v$ with holomorphic parameters $\tau$ defined in a neighborhood of $[U \times B(R)]$. Then, there exists a constant $r_{0}>0$ such that the following hold:

Take arbitrary $h$ and $r$ such that $0<h<h_{0}$ and $0<r<r_{0}$ respectively. Let $\Omega$ be any $h-\Sigma$-flat open convex subset of $V$ with $\operatorname{dia} \Omega \leqslant r$. Then, there exists a constant $C_{A}$ such that for any $s^{\prime}$ and $R^{\prime}$ with $0<s^{\prime}<1$ and $0<R^{\prime} \leqslant R$ respectively,

(1) if $v>0$, then for any $0<s<s^{\prime}$ and $u \in E\left(B\left(R^{\prime}\right) ; X_{s^{\prime}}\right)$ it follows that

$$
\left\|A_{\Sigma} u\right\|_{R^{\prime}, s} \leqslant \frac{C_{A}}{\left(s^{\prime}-s\right)^{v}}\|u\|_{R^{\prime}, s^{\prime}}
$$

(2) if $v \leqslant 0$, then for any $0 \leqslant s<s^{\prime}$ and $u \in E\left(B\left(R^{\prime}\right)\right.$; $\left.X_{s^{\prime}}\right)$ it follows that

$$
\left\|A_{\Sigma} u\right\|_{R^{\prime}, s} \leqslant C_{A}\|u\|_{R^{\prime}, s^{\prime}}
$$

Proof. (1) Since $A$ is defined in a neighborhood of $[U \times B(R)]$, we can find a relatively compact open set $U^{\prime}$ and a vector $R_{0}$ such that $[U] \Subset U^{\prime}$, $R \prec R_{0}$ and $A$ is defined in a neighborhood of $\left[U^{\prime} \times B\left(R_{0}\right)\right]$. Thus, there exist positive constants $K$ and $M$ such that for any $j \leqslant v$

$$
\sup \left\{\left|A_{j}(z, \tau ; \zeta)\right| ;(z, \zeta) \in\left[U^{\prime}\right], \tau \in\left[B\left(R_{0}\right)\right]\right\} \leqslant K M^{v-j}(v-j) !
$$

where $\sum_{j \leqslant v} A_{j}(z, \tau ; \zeta)$ denotes the total symbol of $A\left(z, \tau ; \partial_{z}\right)$ and each $A_{j}(z, \tau ; \zeta)$ is homogeneous of degree $j$ with respect to $\zeta$-variables. Thus by the Taylor expansion we can write 


$$
A_{j}(z, \tau ; \zeta)=\sum_{\beta \in N_{0}{ }^{d}} \tau^{\beta} A_{j}^{(\beta)}(z ; \zeta)
$$

Therefore, by Cauchy's inequality for any $j \leqslant v$ we have

$$
\sup \left\{\left|A_{j}^{(\beta)}(z ; \zeta)\right| ;(z ; \zeta) \in\left[U^{\prime}\right]\right\} \leqslant \frac{K M^{v-j}}{R_{0}^{\beta}}(v-j) !
$$

Set

$$
A^{(\beta)}\left(z ; \partial_{z}\right):=\sum_{j \leqslant v} A_{j}^{(\beta)}\left(z ; \partial_{z}\right)
$$

Then, we easily see that

$$
\begin{aligned}
& A\left(z, \tau ; \partial_{z}\right)=\sum_{\beta \in \boldsymbol{N}_{0^{d}}} \tau^{\beta} A^{(\beta)}\left(z ; \partial_{z}\right), \\
& A^{(\beta)}\left(z ; \partial_{z}\right) \in \Gamma\left(\left[U^{\prime}\right] ; \mathscr{E}_{C^{n}}(v)\right) .
\end{aligned}
$$

Note that taking $0<r_{0}<1 / M$, we can assume that Bony-Schapira's actions of all the microdifferential operators under consideration are well-defined under the assumption of Proposition 2.2 since we can take a constant $M$ in common (see the argument of the proof of Lemma 2.3 below). We remark the following lemma:

2.3. Lemma. (cf. Oaku [O2]). There exists a constant $C$ such that for any $0<s<s^{\prime}<1, \beta \in N_{0}{ }^{d}$ and $v \in X_{s^{\prime}}$, it follows that

$$
\left\|A_{\Sigma}^{(\beta)} v\right\|_{s} \leqslant \frac{C}{\left(s^{\prime}-s\right)^{v} R_{0}{ }^{\beta}}\|v\|_{s^{\prime}} .
$$

Proof of Lemma 2.3. Since ord $\partial_{z_{1}}{ }^{-v} A^{(\beta)} \leqslant 0$, we have

$$
A_{\Sigma}^{(\beta)}=\partial_{z_{1}}{ }^{v}\left(\partial_{z_{1}}{ }^{-v} A^{(\beta)}\right)_{\Sigma}
$$

by Proposition 2.1.2 of Bony-Schapira [Bo-Sc]. Denote the total symbol of $\partial_{z_{1}}{ }^{-v} A^{(\beta)}$ by $\sum_{j \geqslant 0} B_{-j}^{(\beta)}(z ; \zeta)$ where each $B_{-j}(z ; \zeta)$ is homogeneous of degree $-j$ with respect to $\zeta$-variables. Then we may assume that for any $j \geqslant 0$ 


$$
\sup \left\{\left|B_{-j}^{(\beta)}(z ; \zeta)\right| ;(z, \zeta) \in[U]\right\} \leqslant \frac{K M^{j}}{R_{0}{ }^{\beta}} j !
$$

Thus by the proof of Proposition 2.4.3 of [Bo-Sc] we can find a constant $C^{\prime}>0$ (not depending on $s$ and $s^{\prime}$ ) such that for any $z \in \Omega_{s}$ and $\beta \in \mathbb{N}_{0}{ }^{d}$

$$
\left|\left(\partial_{z_{1}}{ }^{-v} A^{(\beta)}\right)_{\Sigma} v(z)\right| \leqslant \frac{K C^{\prime}}{d_{s^{\prime}}^{\prime}(z)^{c_{0}} R_{0}{ }^{\beta}}\|v\|_{s^{\prime}}
$$

Hence, setting $d_{s^{\prime}}^{1}(z):=\inf \left\{\left|z_{1}-w_{1}\right| ; w \in C^{n} \backslash \Omega_{s^{\prime}}, z_{j}=w_{j}(2 \leqslant j \leqslant n)\right\}$ and $z^{\prime}:=\left(z_{2}\right.$, $\ldots, z_{n}$ ) we have

$$
\begin{aligned}
\left|\partial_{z_{1}}{ }^{v}\left(\partial_{z_{1}}{ }^{-v} A^{(\beta)}\right)_{\Sigma} v(z)\right| & =\left|\frac{v !}{2 \pi \sqrt{-1}} \oint_{2\left|z_{1}-w_{1}\right|=d_{s^{\prime}}^{1}(z)} \frac{\left(\partial_{z_{1}}{ }^{-v} A^{(\beta)}\right)_{\Sigma} v\left(w_{1}, z^{\prime}\right)}{\left(w_{1}-z_{1}\right)^{v+1}} d w_{1}\right| \\
& \left.\ll\left(\frac{2}{d_{s^{\prime}}^{1}(z)}\right)^{v} v ! \sup \left\{\mid \partial_{z_{1}}{ }^{-v} A^{(\beta)}\right)_{\Sigma} v\left(w_{1}, z^{\prime}\right)|; 2| z_{1}-w_{1} \mid=d_{s^{\prime}}^{1}(z)\right\} \\
& \ll\left(\frac{2}{d_{s^{\prime}}^{1}(z)}\right)^{v} \frac{K C^{\prime} v !}{R_{0}^{\beta}}\|v\|_{s^{\prime}} \sup \left\{d_{s^{\prime}}^{\prime}\left(w_{1}, z^{\prime}\right)^{-c_{0} ;} ; 2\left|z_{1}-w_{1}\right|=d_{s^{\prime}}^{1}(z)\right\} .
\end{aligned}
$$

Setting $\tilde{w}_{1}:=z_{1}+2\left(w_{1}-z_{1}\right)$ for any $w$ with $2\left|z_{1}-w_{1}\right|=d_{s^{\prime}}^{1}(z)$ we have $\left(\tilde{w}_{1}, z^{\prime}\right) \in\left[\Omega_{s^{\prime}}\right]$. If $2\left|z_{j}-\tilde{w}_{j}\right| \leqslant d_{s^{\prime}}^{\prime}(z)(2 \leqslant j \leqslant n)$, then we have $\left(z_{1}, z^{\prime}+2\left(\tilde{w}^{\prime}-z^{\prime}\right)\right) \in\left[\Omega_{s^{\prime}}\right]$. Therefore by the convexity of $\left[\Omega_{s^{\prime}}\right]$, we have $\left(w_{1}, \tilde{w}^{\prime}\right) \in\left[\Omega_{s^{\prime}}\right]$. Thus it follows that $2 d_{s^{\prime}}^{\prime}\left(w_{1}, z^{\prime}\right) \geqslant d_{s^{\prime}}^{\prime}(z)$. Hence

$$
\begin{aligned}
\left|A_{\Sigma}^{(\beta)} v(z)\right| & =\left|\partial_{z_{1}}{ }^{v}\left(\partial_{z_{1}}{ }^{-v} A^{(\beta)}\right)_{\Sigma} v(z)\right| \\
& \leqslant \frac{2^{v+c_{0}} K C^{\prime} v !}{d_{s^{\prime}}^{\prime}(z)^{c_{0}} d_{s^{\prime}}^{1}(z)^{v} R_{0}{ }^{\beta}}\|v\|_{s^{\prime}}
\end{aligned}
$$

Setting $c^{\prime}:=\operatorname{dis}\left(z_{0}, C^{n} \backslash \Omega\right)$, we easily see that $\operatorname{dis}\left(\Omega_{s}, C^{n} \backslash \Omega_{s^{\prime}}\right) \geqslant c^{\prime}\left(s^{\prime}-s\right)$. Therefore it follows that for any $z \in \Omega_{s}$

$$
\begin{aligned}
\left|A_{\Sigma}^{(\beta)} v(z)\right| & \leqslant \frac{2^{v+c_{0}} K C^{\prime} v !}{d_{s^{\prime}}^{\prime}(z)^{c_{0}}\left(c^{\prime}\left(s^{\prime}-s\right)\right)^{v} R_{0}{ }^{\beta}}\|v\|_{s^{\prime}} \\
& \leqslant \frac{2^{v+c_{0}} K C^{\prime} v !}{d_{s}^{\prime}(z)^{c_{0}}\left(c^{\prime}\left(s^{\prime}-s\right)\right)^{v} R_{0}{ }^{\beta}}\|v\|_{s^{\prime}} .
\end{aligned}
$$

Hence we may take 


$$
C:=\frac{2^{v+c_{0}} K C^{\prime} v !}{\left(c^{\prime}\right)^{v}}
$$

End of Proof of Proposition 2.2. By definition, for any $u(z, \tau)=\sum_{\gamma \in \mathbb{N}_{0}{ }^{d}} u_{\gamma}(z) \tau^{\gamma}$ $\in E\left(B\left(R^{\prime}\right) ; X_{s^{\prime}}\right)$ we have

$$
A_{\Sigma} u(z, \tau)=\sum_{\beta . \gamma \in N_{0}{ }^{d}}\left(A_{\Sigma}^{(\beta)} u_{\gamma}\right)(z) \tau^{\beta+\gamma}
$$

formally. Hence remarking

$$
\Phi_{R_{0}}(T) \Phi_{R^{\prime}}(T) \ll \Phi_{R_{0}}\left(R^{\prime}\right) \Phi_{R^{\prime}}(T) \ll \Phi_{R_{0}}(R) \Phi_{R^{\prime}}(T),
$$

by Lemma 2.3 we have

$$
\begin{aligned}
A_{\Sigma} u(z, T) & =\sum_{\beta, \gamma \in N_{0} d^{d}}\left(A_{\Sigma}^{(\beta)} u_{\gamma}\right)(z) T^{\beta+\gamma} \\
& \ll \sum_{\beta \in N_{0} d} \frac{C T^{\beta}}{\left(s^{\prime}-s\right)^{\nu} R_{0}^{\beta}} \sum_{\gamma \in N_{0}^{d}}\left\|u_{\gamma}\right\|_{s^{\prime}} T^{\gamma} \\
& \ll \frac{C \Phi_{R_{0}}(T)}{\left(s^{\prime}-s\right)^{v}}\|u\|_{R^{\prime}, s^{\prime}} \Phi_{R^{\prime}}(T) \\
& \ll \frac{C \Phi_{R_{0}}(R)}{\left(s^{\prime}-s\right)^{v}}\|u\|_{R^{\prime}, s^{\prime}} \Phi_{R^{\prime}}(T) .
\end{aligned}
$$

Thus we may set

$$
C_{A}:=C \Phi_{R_{0}}(R)
$$

Proof of (2) is similar.

2.4. Remark. For the argument to the proof of Proposition 2.2, we may assume that if we take $r_{0}>0$ small enough, then Bony-Schapira's actions of finite numbers of microdifferential operators under consideration are well-defined on $\Omega$ under the following assumption:

$\Omega$ is an $h$ - $\Sigma$-flat open convex subset of $V$ with dia $\Omega \leqslant r$ for some $h$ and $r$ such that $0<h<h_{0}$ and $0<r<r_{0}$.

Thus hereafter, we often omit the argument that Bony-Schapira's action 
of a microdifferential operator in question is well-defined.

\section{§3. Proof of Main Theorem}

In this section, we prove Theorem 2.1. Note that our method of proof is similar to that of [M] and [O2]. We inherit the notation and assumption of preceding section.

First, we prove the following:

3.1. Proposition. There exists a constant $r_{0}>0$ such that for any constants $h$ and $r$ with $0<r<r_{0}$ and $0<h<h_{0}$, the following hold:

Let $\Omega$ be any $h-\Sigma$-flat open convex subset of $V$ such that dia $\Omega \leqslant r$. Then there exists a linear operator $\mathscr{H}_{Q}$ such that

(1) for any $R^{\prime}, s$ and $\alpha$ such that $0<R^{\prime} \leqslant R, 0<s<1$ and $0 \leqslant \alpha^{(v)} \leqslant m^{(v)}$ $(1 \leqslant v \leqslant N)$

$$
\left(\begin{array}{ccc}
\left(\partial_{\tau} \tau\right)^{\alpha^{(1)}} & & 0 \\
0 & \ddots & \\
0 & & \left(\partial_{\tau} \tau\right)^{\alpha^{(N)}}
\end{array}\right) \cdot \mathscr{H}_{Q}: E\left(B\left(R^{\prime}\right) ; X_{s}\right)^{\oplus N} \rightarrow E\left(B\left(R^{\prime}\right) ; X_{s}\right)^{\oplus N} .
$$

Further there exists constant $C_{Q}$ (not depending on $R^{\prime}, s$ and $\left.\alpha^{(v)}\right)$ such that

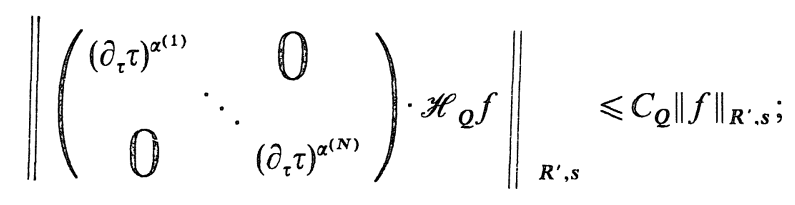

(2) $Q_{\Sigma} \cdot \mathscr{H}_{Q}=\mathscr{H}_{Q} \cdot Q_{\Sigma}=\mathrm{id}: E\left(B\left(R^{\prime}\right) ; X_{s}\right)^{\oplus N} \rightarrow E\left(B\left(R^{\prime}\right) ; X_{s}\right)^{\oplus N}$.

Proof. Take a relatively open set $W^{\prime}$ such that $[W] \Subset W^{\prime}$ and $Q$ is defined on $\left[W^{\prime}\right]$. Shrinking $W$ and $W^{\prime}$ if necessary, we may assume that for any $(z ; \zeta) \in\left[W^{\prime}\right]$ and $\beta \in \mathbb{N}_{0}{ }^{d}$

$$
\left|\mathscr{I}_{Q}(z ; \zeta ; \beta)\right| \geqslant C \prod_{v=1}^{N}\left(\beta+\mathbb{1}_{d}\right)^{m^{(v)}}
$$

There exist positive constants $K$ and $M$ such that for all $j \geqslant 0,1 \leqslant \mu, v \leqslant N$ and $0 \leqslant \alpha \leqslant m^{(v)}$

$$
\sup \left\{\left|Q_{\alpha,-j}^{(\mu, v)}(z ; \zeta)\right| ;(z ; \zeta) \in\left[W^{\prime}\right]\right\} \leqslant K M^{j} j !
$$


where $\sum_{j \geqslant 0} Q_{\alpha,-j}^{(\mu, v)}(z ; \zeta)$ denotes the total symbol of $Q_{\alpha}^{(\mu, v)}$ and each $Q_{\alpha,-j}^{(\mu, v)}(z ; \zeta)$ is homogeneous of degree $-j$ with respect to $\zeta$-variables. For any $f(z, \tau)$, $u(z, \tau) \in E\left(B(R) ; X_{s}\right)^{\oplus N}$, we write $f(z, \tau)=\sum_{\beta} f_{\beta}(z) \tau^{\beta}$ and $u(z, \tau)=\sum_{\beta} u_{\beta}(z) \tau^{\beta}$ respectively. For any $\beta \in \mathbb{N}_{0}{ }^{d}$ we define $\widetilde{Q}_{\beta}\left(z ; \partial_{z}\right) \in \operatorname{Mat}\left(N ; \Gamma\left(\left[W^{\prime}\right] ; \mathscr{E}_{C^{n}}(0)\right)\right)$ by

$$
\widetilde{Q}_{\beta}^{(\mu, v)}\left(z ; \partial_{z}\right):=\sum_{0 \leqslant \alpha \leqslant m^{(v)}} Q_{\alpha}^{(\mu, v)}\left(z ; \partial_{z}\right) \mathscr{I}_{\alpha}(\beta) \in \Gamma\left(\left[W^{\prime}\right] ; \mathscr{E}_{\mathbf{C}^{n}}(0)\right)
$$

Then, the formal calculation implies that

$$
\left(Q\left(z, \tau ; \partial_{z}, \partial_{\tau}\right)_{\Sigma} u(z, \tau)\right)_{\mu}=\sum_{\beta \in N_{0}{ }^{d}} \sum_{\nu=1}^{N} \tilde{Q}_{\beta}^{(\mu, v)}\left(z ; \partial_{z}\right)_{\Sigma} u_{v, \beta}(z) \tau^{\beta} .
$$

Thus the equation $Q_{\Sigma} u=f$ is equivalent to

$$
\sum_{v=1}^{N} \widetilde{Q}_{\beta}^{(\mu, v)} u_{v, \beta}=f_{\mu, \beta}
$$

for any $\mu$ and $\beta$. Since $\operatorname{det}\left(\sigma_{0}\left(\widetilde{Q}_{\beta}^{(\mu, v)}\right)(z ; \zeta)\right)=\mathscr{I}_{Q}(z ; \zeta ; \beta)$, the assumption (3.2) implies that each $\tilde{Q}_{\beta}\left(z ; \partial_{z}\right)$ is invertible on $\left[W^{\prime}\right]$. Set $S_{\beta}\left(z ; \partial_{z}\right):=\widetilde{Q}_{\beta}\left(z ; \partial_{z}\right)^{-1}$ $\in \operatorname{Mat}\left(N ; \Gamma\left(\left[W^{\prime}\right] ; \mathscr{E}_{C^{n}}(0)\right)\right)$.

3.2. Lemma. There exist constants $K_{1}$ and $M_{1}$ such that for all $j \geqslant 0$, $1 \leqslant \mu, v \leqslant N$ and $\beta \in N_{0}{ }^{d}$

$$
\sup \left\{\left|S_{\beta,-j}^{(\mu, v)}(z ; \zeta)\right| ;(z ; \zeta) \in[W]\right\} \leqslant \frac{K_{1}}{\left(\beta+\mathbb{1}_{d}\right)^{m^{(\mu)}}} M_{1}{ }^{j} j ! .
$$

Here $\sum_{j \geqslant 0} S_{\beta,-j}^{(\mu, v)}(z ; \zeta)$ denotes the total symbol of $S_{\beta}^{(\mu, v)}\left(z ; \partial_{z}\right)$ and each $S_{\beta,-j}^{(\mu, v)}(z ; \zeta)$ is homogeneous of degree $-j$ with respect to $\zeta$-variables.

Proof of Lemma 3.2. Set $\widetilde{R}_{\beta}(z ; \zeta):=\sigma_{0}\left(\tilde{Q}_{\beta}\right)(z ; \zeta)^{-1}$. There exists a constant $\tilde{C}>0$ such that for any $0 \leqslant \alpha \leqslant m^{(v)}$ and $\beta \in N_{0}{ }^{d}$

$$
\left|\mathscr{I}_{\alpha}(\beta)\right| \leqslant \widetilde{C}\left(\beta+\mathbb{1}_{d}\right)^{m^{(v)}} .
$$

Let $\Delta^{(\mu, v)}(z ; \zeta)$ be the $(\mu ; v)$-th cofactor of $\sigma_{0}\left(\tilde{Q}_{\beta}\right)(z ; \zeta)$. Then by (3.4) and (3.7) there exists a constant $C^{\prime}>0$ such that 


$$
\sup \left\{\left|\Delta^{(\mu, v)}(z ; \zeta)\right| ;(z ; \zeta) \in\left[W^{\prime}\right]\right\} \leqslant \prod_{l \neq v} C^{\prime}\left(\beta+\mathbb{1}_{d}\right)^{m^{(l)}}
$$

Hence by the assumption (3.2),

$$
\sup \left\{\left|\tilde{R}_{\beta}^{(\mu, v)}(z ; \zeta)\right| ;(z ; \zeta) \in\left[W^{\prime}\right]\right\} \leqslant \frac{C^{\prime}}{C\left(\beta+\mathbb{1}_{d}\right)^{m^{(\mu)}}}
$$

Now, for a microdifferential operator $A\left(z ; \partial_{z}\right)$ of order zero defined in a neigh-borhood of $[W]$ with the total symbol $\sum_{j \geqslant 0} A_{-j}\left(z ; \partial_{z}\right)$ where each $A_{-j}(z ; \zeta)$ is homogeneous of degree $-j$ with respect to $\zeta$-variables, $N_{0}^{[W]}(A ; \rho)$ denotes the formal norm in the sense of L. Boutet de Monvel and P. Krée [Bou-K]; that is,

$$
N_{0}^{[W]}(A ; \rho):=\sum_{\substack{0 \leq \tilde{\alpha} \in j \\ \tilde{\alpha} \in N_{0^{d}}}} \frac{2(2 n)^{-j} j !}{(|\tilde{\alpha}|+j) !(|\widetilde{\beta}|+j) !} \sup \left\{\left|\partial_{z}^{\tilde{\alpha}} \partial_{\zeta^{\beta}}^{\tilde{\beta}} A_{-j}(z ; \zeta)\right| ;(z ; \zeta) \in[W]\right\} \rho^{2 j+|\tilde{\alpha}+\tilde{\beta}|}
$$

where $\rho$ is a parameter.

3.3. Sublemma. There exist positive constants $L_{1}$ and $c_{1}$ such that

$$
N_{0}^{[W]}\left(\tilde{R}_{\beta}^{(\mu, v)} ; \rho\right) \ll \sum_{j=0}^{\infty} \frac{L_{1}}{\left(\beta+\mathbb{1}_{d}\right)^{m^{(\mu)}}}\left(\frac{\rho}{c_{1}}\right)^{j}
$$

Proof of Sublemma 3.3. By Cauchy's inequality and (3.8), we have

$$
\sup \left\{\left|\partial_{z}^{\tilde{\alpha}} \partial_{\zeta}^{\widetilde{\beta}} \tilde{R}_{\beta}(z ; \zeta)\right| ;(z ; \zeta) \in[W]\right\} \leqslant \frac{C^{\prime} \tilde{\alpha} ! \tilde{\beta} !}{C\left(\beta+\mathbb{1}_{d}\right)^{m^{(\mu)}}}\left(\frac{1}{c^{\prime}}\right)^{|\tilde{\alpha}+\tilde{\beta}|}
$$

where $c^{\prime}$ is a some constant depending only on $W$ and $W^{\prime}$. Thus it follows that

$$
\begin{aligned}
N_{0}^{[W]}\left(\tilde{R}_{\beta}^{(\mu, v)} ; \rho\right) & \left.\ll \sum_{\tilde{\alpha}, \tilde{\beta}|\tilde{\alpha}| !|\tilde{\beta}| !} \frac{2 \tilde{\alpha} ! \tilde{\beta} !}{c^{\prime}}\right)^{|\tilde{\alpha}+\tilde{\beta}|} \frac{C^{\prime}}{C\left(\beta+\mathbb{1}_{d}\right)^{m^{(\mu)}}} \\
& \ll \sum_{j=0}^{\infty}\left(\begin{array}{c}
j+2 n-1 \\
j
\end{array}\right)\left(\frac{\rho}{c^{\prime}}\right)^{j} \frac{2 C^{\prime}}{C\left(\beta+\mathbb{1}_{d}\right)^{m^{(\mu)}}} \\
& \ll \sum_{j=0}^{\infty}\left(\frac{2 \rho}{c^{\prime}}\right)^{j} \frac{2^{2 n} C^{\prime}}{C\left(\beta+\mathbb{1}_{d}\right)^{m^{(\mu)}}} .
\end{aligned}
$$


Thus we may set $L_{1}:=2^{2 n} C^{\prime} / C$ and $c_{1}:=c^{\prime} / 2$.

Proof of Lemina 3.2 continued. Next, set

$$
R_{\beta}\left(z ; \partial_{z}\right):=I_{N}-\tilde{Q}_{\beta}\left(z ; \partial_{z}\right) \tilde{R}_{\beta}\left(z ; \partial_{z}\right) \in \operatorname{Mat}\left(N ; \Gamma\left(\left[W^{\prime}\right] ; \mathscr{E}_{C^{n}}(-1)\right)\right),
$$

where $I_{N}$ denotes the identity matrix of size $N \times N$. Therefore denoting the total symbol of $R_{\beta}^{(\mu, v)}\left(z ; \partial_{z}\right)$ by $\underset{j>0}{\sum} R_{\beta,-j}^{(\mu, v)}(z ; \zeta)$ where each $R_{\beta,-j}^{(\mu, v)}(z ; \zeta)$ is homogeneous of degree $-j$ with respect to $\zeta$-variables, for any $j>0$ we have

$$
R_{\beta,-j}^{(\mu, v)}(z ; \zeta)=-\sum_{\substack{j=p+|\gamma| \\ p \geqslant 0 .|\gamma|>0}} \sum_{l=1}^{N} \frac{1}{\gamma !} \partial_{\zeta^{\gamma}}\left(\sum_{0 \leqslant \alpha \leqslant m^{(l)}} Q_{\alpha,-p}^{(\mu, l)}(z ; \zeta) \mathscr{I}_{\alpha}(\beta)\right) \cdot \partial_{z}{ }^{\gamma} \tilde{R}_{\beta}^{(l, v)}(z ; \zeta) .
$$

There exist constants $K^{\prime}>0$ and $L^{\prime}>0$ such that for all $j \geqslant 0$

$$
\sup \left\{\left|\sum_{0 \leqslant \alpha \leqslant m^{(v)}} Q_{\alpha,-j}^{(\mu, v)}(z ; \zeta)\right| ;(z ; \zeta) \in\left[W^{\prime}\right]\right\} \leqslant K^{\prime} M^{j} j !
$$

3.4. Sublemma. There exist positive constants $L_{2}$ and $c_{2}$ such that

$$
N_{0}^{[W]}\left(R_{\beta}^{(\mu, v)} ; \rho\right) \ll L_{2} \sum_{p=2}^{\infty}\left(\frac{\rho}{c_{2}}\right)^{p} .
$$

Proof of Sublemma 3.4. Take $W \Subset W^{\prime \prime} \Subset W^{\prime} . \quad$ By (3.8), (3.9) and Cauchy's inequality we have

$$
\begin{array}{r}
\sup \left\{\left|\partial_{z}^{\gamma} \tilde{R}_{\beta}^{(\mu, v)}(z ; \zeta)\right| ;(z ; \zeta) \in\left[W^{\prime \prime}\right]\right\} \leqslant \frac{C^{\prime} \gamma !}{C\left(\beta+\mathbf{1}_{d}\right)^{m^{(\mu)}}}\left(\frac{1}{c^{\prime \prime}}\right)^{|\gamma|}, \\
\sup \left\{\left|\partial_{\zeta^{\gamma}}\left(\sum_{0 \leqslant \alpha \leqslant m^{(v)}} Q_{\alpha .-j}^{(\mu, v)}\right)(z ; \zeta)\right| ;(z ; \zeta) \in\left[W^{\prime \prime}\right]\right\} \leqslant \gamma !\left(\frac{1}{c^{\prime \prime}}\right)^{|\gamma|} K^{\prime} M^{j} j ! .
\end{array}
$$

Thus it follows that for any $j>0$

$$
\begin{aligned}
\sup \left\{\left|R_{\beta,-j}^{(\mu, v)}(z ; \zeta)\right| ;(z ; \zeta) \in\left[W^{\prime \prime}\right]\right\} & \leqslant \sum_{\substack{j=p+|\gamma| \\
p \geqslant 0}} \gamma !\left(\frac{1}{\left(c^{\prime \prime}\right)^{2}}\right)^{|\gamma|} N K^{\prime} L_{1} M^{p} p ! \\
& \leqslant N K^{\prime} L_{1} \sum_{\substack{j=p+|\gamma| \\
p \geqslant 0}}\left(\frac{M}{\left(c^{\prime \prime}\right)^{2}}\right)^{j} j !
\end{aligned}
$$




$$
\leqslant 2^{n-1} N K^{\prime} \mathbb{L}_{1}\left(\frac{2 M}{\left(c^{\prime \prime}\right)^{2}}\right)^{j} j !
$$

Therefore, setting $L^{\prime \prime}:=2^{n-1} N K^{\prime} L_{1}$ we have

$$
\sup \left\{\left|\partial_{z}^{\tilde{\alpha}} \partial_{\zeta}{ }_{\zeta}^{\tilde{\beta}} R_{\beta,-j}^{(\mu, v)}(z ; \zeta)\right| ;(z ; \zeta) \in[W]\right\} \leqslant \tilde{\alpha} ! \tilde{\beta} ! L^{\prime \prime}\left(\frac{1}{c^{\prime \prime \prime}}\right)^{|\tilde{\alpha}+\tilde{\beta}|}\left(\frac{2 M}{\left(c^{\prime \prime}\right)^{2}}\right)^{j} j !
$$

Since we may assume that $c^{\prime \prime \prime} \leqslant 1$ and $\left(c^{\prime \prime}\right)^{2} \leqslant 2 M$, we obtain

$$
\begin{aligned}
N_{0}^{[W]}\left(R_{\beta}^{(\mu, v)} ; \rho\right) & \ll \sum_{\substack{\tilde{\alpha}, \tilde{\beta} \geqslant 0 \\
j \geqslant 1}} \frac{L^{\prime \prime} \tilde{\alpha} ! \tilde{\beta} !(j !)^{2}}{n(|\tilde{\alpha}|+j) !(|\widetilde{\beta}|+j) !}\left(\frac{1}{c^{\prime \prime \prime}}\right)^{|\tilde{\alpha}+\widetilde{\beta}|}\left(\frac{2 M}{\left(c^{\prime \prime}\right)^{2}}\right)^{j} \rho^{2 j+|\tilde{\alpha}+\widetilde{\beta}|} \\
& \ll \frac{L^{\prime \prime}}{n} \sum_{p=|\tilde{\alpha}+\widetilde{\beta}|+2 j \geqslant 2}\left(\frac{1}{c^{\prime \prime \prime}}\right)^{|\tilde{\alpha}+\widetilde{\beta}|}\left(\frac{2 M}{\left(c^{\prime \prime}\right)^{2}}\right)^{j} \rho^{p} \\
& \ll \frac{2^{2 n} L^{\prime \prime}}{n} \sum_{p=2}^{\infty}\left(\frac{2 M}{\left(c^{\prime \prime}\right)^{2} c^{\prime \prime \prime}}\right)^{p} \rho^{p} .
\end{aligned}
$$

Thus we may set $L_{2}:=2^{2 n} L^{\prime \prime} / n$ and $c_{2}:=\left(c^{\prime \prime}\right)^{2} c^{\prime \prime \prime} /(2 M)$.

End of Proof of Lemma 3.2. By Sublemmas 3.3 and 3.4, we see that if $\rho_{0}$ is a sufficiently small positive constant, then

$$
\left\{\begin{array}{l}
N_{0}^{[W]}\left(\tilde{\mathbb{R}}_{\beta}^{(\mu, v)} ; \rho_{0}\right) \leqslant \frac{2 L}{\left(\beta+\mathbb{1}_{d}\right)^{m^{(\mu)}}} \\
N_{0}^{[W]}\left(\mathbb{R}_{\beta}^{(\mu, v)} ; \rho_{0}\right) \leqslant \frac{1}{2 N}
\end{array}\right.
$$

Since we see that $S_{\beta}=\sum_{j=0}^{\infty} \widetilde{R}_{\beta} R_{\beta}^{j}$, setting $K_{1}:=4 L$, by (3.10) we have

$$
N_{0}^{[W]}\left(S_{\beta} ; \rho_{0}\right) \leqslant \sum_{j=0}^{\infty}\left(\frac{1}{2}\right)^{j} \frac{2 \mathbb{L}}{\left(\beta+\mathbb{1}_{d}\right)^{m^{(\mu)}}}=\frac{K_{1}}{\left(\beta+\mathbb{1}_{d}\right)^{m^{(\mu)}}} .
$$

Thus setting $M_{1}:=2 n /\left(\rho_{0}{ }^{2}\right)$ we have

$$
\sup \left\{\left|S_{\beta,-j}^{(\mu, v)}(z ; \zeta)\right| ;(z ; \zeta) \in[W]\right\} \leqslant \frac{K_{1}}{\left(\beta+\mathbb{1}_{d}\right)^{m^{(\mu)}}}\left(\frac{2 n}{\rho_{0}^{2}}\right)^{j} j !
$$




$$
=\frac{K_{1}}{\left(\beta+\mathbb{1}_{d}\right)^{m^{(\mu)}}} M_{1}^{j} j !
$$

Hence the proof of Lemma 3.2 is completed.

End of Proof of Proposition 3.1. Let us take $r_{0} \leqslant \min \left\{M^{-1}, M_{1}^{-1}\right\}$. Then, by Lemma 3.2 we see that $Q_{\beta_{\Sigma}}$ and $S_{\beta_{\Sigma}}$ are well-defined on $\Omega$ with $\operatorname{dia} \Omega<r_{0}$. Moreover, the equation $\sum_{\nu=1}^{N} \widetilde{Q}_{\beta}^{(\mu, v)}{ }_{\Sigma} u_{v, \beta}=f_{\mu, \beta}$ is equivalent to $u_{\mu, \beta}=$ $\sum_{v=1}^{N} S_{\beta}^{(\mu, v)} f_{v, \beta}$. Thus if we set $\mathscr{H}_{Q}$ by

$$
\left(\mathscr{H}_{Q} f\right)_{\mu}(z, \tau):=\sum_{\nu=1}^{N} \sum_{\beta \in N_{0^{d}}} S_{\beta}^{(\mu, v)} f_{v, \beta}(z) \tau^{\beta}
$$

then we have

$$
\left(\partial_{\tau} \tau\right)^{\alpha^{(\mu)}}\left(\mathscr{H}_{Q} f_{\mu}\right)(z, \tau)=\sum_{\beta \in N_{0} d}\left(\beta+\mathbb{1}_{d}\right)^{\alpha^{(\mu)}} S_{\beta}^{(\mu, v)} f_{v, \beta}(z) \tau^{\beta}
$$

Thus we easily see that all the properties are satisfied.

Next, we prove the following:

3.5. Proposition. There exist constants $r_{0}>0$ and $\stackrel{\circ}{R}$ with $0<\stackrel{\circ}{R} \leqslant R$ such that for any constants $h$ and $r$ with $0<r<r_{0}$ and $0<h<h_{0}$, the following hold:

Let $\Omega$ be any $h-\Sigma$-flat open convex subset of $V$ such that dia $\Omega \leqslant r$. Then, for any $R^{\prime}$ and such that $0<R^{\prime} \leqslant R^{\circ}$ and $0<s<1$, the following equation is uniquely solvable on $E\left(B\left(R^{\prime}\right) ; X_{s}\right)$ :

$$
\left(Q+Q^{\prime}\right)_{\Sigma} u=f
$$

Precisely, the operator $\left(Q+Q^{\prime}\right)_{\Sigma}$ is invertible on $E\left(B\left(R^{\prime}\right) ; X_{s}\right)$.

Proof. We use the notation of the proof of Proposition 3.1. For any $f, u \in E\left(B(R) ; X_{s}\right)^{\oplus N}$, we write $f(z, \tau)=\sum_{\beta} f_{\beta}(z) \tau^{\beta}$ and $u(z, \tau)=\sum_{\beta} u_{\beta}(z) \tau^{\beta}$ respectively. Thus, by Proposition 3.1, the equation (3.11) is equivalent to 


$$
\left(I+Q_{\Sigma}^{\prime} \mathscr{H}_{Q}\right) Q_{\Sigma} u=f
$$

Set $v:=Q_{\Sigma} u \in E\left(B(R) ; X_{s}\right)^{\oplus N}$. Since

$$
\left(Q_{\Sigma}^{\prime} \mathscr{H}_{Q} v\right)_{\mu}=\sum_{l, v=1}^{N} \sum_{0 \leqslant \alpha \leqslant m^{(l)}} \sum_{i=1}^{d} \tau_{i} Q_{i, \alpha}^{(\mu . l)} \Sigma\left(\partial_{\tau} \tau\right)^{\alpha} \mathscr{H}_{Q}^{(l, v)} v_{v}
$$

by Propositions 2.2 and 3.1 , there exist constants $C_{Q}$ and $C_{i, \alpha}^{(l, v)}$ such that

$$
\left\|\left(Q_{\Sigma^{\prime}}^{\prime} \mathscr{H}_{Q} v\right)_{\mu}\right\|_{R^{\prime}, s} \leqslant C_{Q} \sum_{l, v=1}^{N} \sum_{0 \leqslant \alpha \leqslant m^{(l)}} \sum_{i=1}^{d} R_{i}^{\prime} C_{i, \alpha}^{(l, v)}\left\|v_{v}\right\|_{R^{\prime}, s} .
$$

Thus, if we choose $\stackrel{R}{R}$ as small as

$$
C_{Q} \sum_{\mu, v=1}^{N} \sum_{0 \leqslant \alpha \leqslant m^{(\mu)}} \sum_{i=1}^{d} R_{i}^{\prime} C_{i, \alpha}^{(\mu, v)} \leqslant C_{Q} \sum_{\mu, v=1}^{N} \sum_{0 \leqslant \alpha \leqslant m^{(\mu)}} \sum_{i=1}^{d} \stackrel{\circ}{R}_{i} C_{i, \alpha}^{(\mu, v)}<1,
$$

we can see that $I+Q_{\Sigma}^{\prime} \mathscr{H}_{Q}$ is invertible on $E\left(B\left(R^{\prime}\right) ; X_{s}\right)$. Hence we see that $\mathscr{H}_{Q+Q^{\prime}}:=\mathscr{H}_{Q}\left(I+Q_{\Sigma}^{\prime} \mathscr{H}_{Q}\right)^{-1}$ is the inverse of $\left(Q+Q^{\prime}\right)_{\Sigma}$.

Now, we are ready to prove Theorem 2.1. Choose $\stackrel{R}{ }$ as in Proposition 3.5 and take $0<\tilde{R} \leqslant \AA$. Recall that the equation under consideration is the following:

$$
\left(Q+Q^{\prime}+A\right)_{\Sigma} u=f \in \mathcal{O}\left(B(\tilde{R}) ; X_{s_{0}}\right)^{\oplus N}
$$

First we will prove the existence of a solution $u(z, \tau)$. By Proposition 3.5, the problem is equivalent to

$$
u+\mathscr{H}_{Q+Q^{\prime}} A_{\Sigma} u=\mathscr{H}_{Q+Q^{\prime}} f
$$

(recall that $\left.\mathscr{H}_{Q+Q^{\prime}}=\left(\left(Q+Q^{\prime}\right)_{\Sigma}\right)^{-1}\right)$. Set

$$
u_{p}:= \begin{cases}\mathscr{H}_{Q+Q^{\prime}} f & (p=0), \\ \mathscr{H}_{Q+Q^{\prime}}\left(f-A_{\Sigma} u_{p-1}\right) & (p \in N) .\end{cases}
$$

Moreover, set 


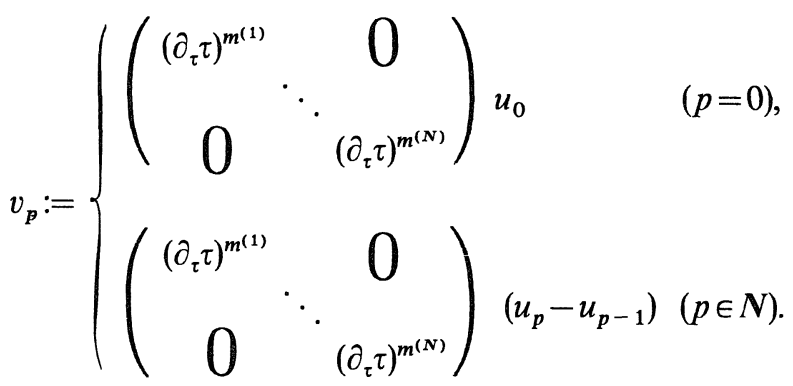

Then we easily see that $v_{0} \in E\left(B\left(R^{\prime}\right) ; X_{s_{0}}\right)^{\oplus N}$ and $v_{p} \in E\left(B\left(R^{\prime}\right) ; X_{s}\right)^{\oplus N}(p \in N)$ for any $0<s<s_{0}$ and $0<R^{\prime} \prec \tilde{R}$. Since each $\left(\partial_{\tau} \tau\right)^{m^{(v)}-\alpha}\left(0 \leqslant \alpha<m^{(v)}\right)$ satisfies the assumption of Proposition 3.1 in the case where $N=1$, we can write

$$
\left(\partial_{\tau} \tau\right)^{\alpha}=\mathscr{H}^{m^{(v)}-\alpha}\left(\partial_{\tau} \tau\right)^{m^{(v)}}, \text { where } \mathscr{H}^{m^{(v)}-\alpha}:=\mathscr{H}_{\left(\partial_{\tau} \tau\right)^{m(v)-\alpha}}
$$

Hence for $0 \leqslant \alpha<m^{(v)}$ we have $\left(\partial_{\tau} \tau\right)^{\alpha} v_{p, v}=\mathscr{H}^{m^{(v)}-\alpha} v_{p, v}$. Thus it follows that

$$
\begin{aligned}
v_{p, \mu} & =-\left(\partial_{\tau} \tau\right)^{m^{(\mu)}} \sum_{l, v=1}^{N} \sum_{0 \leqslant \alpha \leqslant m^{(v)}} \mathscr{H}_{Q+Q^{\prime}}^{(\mu, l)} \tau^{\mathbf{1}_{d}} A_{\alpha}^{(l, v)}\left(z, \tau ; \partial_{z}\right)_{\Sigma}\left(\partial_{\tau} \tau\right)^{\alpha} v_{p-1, v} \\
& =-\left(\partial_{\tau} \tau\right)^{m^{(\mu)}} \sum_{l, v=1}^{N} \sum_{0 \leqslant \alpha \leqslant m^{(v)}} \mathscr{H}_{Q+Q^{\prime}}^{(\mu, l)} \tau^{\mathbf{1}_{d}} A_{\alpha}^{(l, v)}\left(z, \tau ; \partial_{z}\right)_{\Sigma} \mathscr{H}^{m(v)-\alpha} v_{p-1, v}
\end{aligned}
$$

By Propositions 2.2 and 3.5 there exists a constant $M_{1}$ such that for any $0<s \leqslant s_{0}$ and $0 \prec R^{\prime \prime} \leqslant R^{\prime}$ it follows that for any $v \in E\left(B\left(R^{\prime \prime}\right) ; X_{s}\right)^{\oplus N}$

$$
\begin{aligned}
& \left\|\left(\begin{array}{ccc}
\left(\partial_{\tau} \tau\right)^{m^{(1)}} & & 0 \\
& \ddots & \\
0 & & \left(\partial_{\tau} \tau\right)^{m^{(N)}}
\end{array}\right) \mathscr{H}_{Q+Q^{\prime} v}\right\|_{R^{\prime \prime}, s} \\
& =\left\|\left(\begin{array}{ccc}
\left(\partial_{\tau} \tau\right)^{m^{(1)}} & & 0 \\
& \ddots & \\
0 & & \left(\partial_{\tau} \tau\right)^{m^{(N)}}
\end{array}\right) \mathscr{H}_{Q}\left(I+Q_{\Sigma}^{\prime} \mathscr{H}_{Q}\right)^{-1} v\right\|_{R^{\prime \prime}, s} \\
& \leqslant M_{1}\|v\|_{R^{\prime \prime}, s} .
\end{aligned}
$$


We will prove that there exists a constant $M_{2}$ such that for any $0<s<s_{0}$ and $0<R^{\prime \prime} \leqslant R^{\prime}$ we have

$$
\left\|v_{p}\right\|_{R^{\prime \prime}, s} \leqslant M_{1}\|f\|_{R^{\prime}, s_{0}}\left(\frac{M_{1} M_{2} \prod_{j=1}^{d} R_{j}^{\prime \prime}}{\left(s_{0}-s\right)^{|m|}}\right)^{p} .
$$

Since $v_{0} \in E\left(B\left(R^{\prime}\right) ; X_{s_{0}}\right)^{\oplus N}$, by (3.17) we have

$$
\left\|v_{0}\right\|_{R^{\prime \prime}, s} \leqslant\left\|v_{0}\right\|_{R^{\prime}, s_{0}} \leqslant M_{1}\|f\|_{R^{\prime}, s_{0}} .
$$

Thus we have (3.18)-0. By Proposition 2.2, for any $s<s^{\prime}<s_{0}$ and $0 \leqslant \alpha<m^{(v)}$ we have



where each $C_{\alpha}^{(l, v)}$ is a constant not depending on $s, s^{\prime}$ and $R^{\prime \prime}$. Hence we obtain

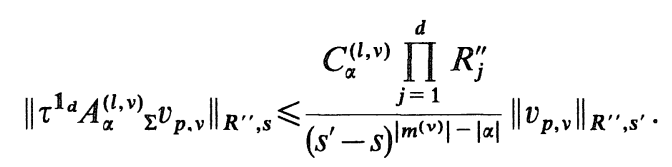

Note the following lemma:

3.6. Lemma. The following equality holds:

$$
\mathscr{H}^{m^{(v)}-\alpha} v_{p, v}(z, \tau)=\int_{[0,1]^{|m(v)|-|\alpha|}} v_{p, v}(z, \sigma \tau) d \sigma
$$

where $\sigma:=\left(\sigma_{1,1}, \ldots, \sigma_{1, m_{1}^{(v)}-\alpha_{1}}, \ldots, \sigma_{d, 1}, \ldots, \sigma_{d, m_{d}^{(v)}-\alpha_{d}}\right) \in[0,1]^{\left|m^{(v)}\right|-|\alpha|}$

$$
\sigma \tau:=\left(\prod_{j=1}^{m_{1}^{(v)}-\alpha_{1}} \sigma_{1, j} \tau_{1}, \ldots, \prod_{j=1}^{m_{d}^{(\nu)}-\alpha_{d}} \sigma_{d, j} \tau_{d}\right) \text { and } d \sigma:=\prod_{i=1}^{d} \prod_{j=1}^{m_{i}^{(\nu)}-\alpha_{2}} d \sigma_{i, j} .
$$

Proof of Lemma 3.6. We have only to remark that for any $\gamma \in \mathbb{N}_{0}{ }^{d}$

$$
\int_{[0,1]^{|m(v)|-|\alpha|}}(\sigma \tau)^{\gamma} d \sigma=\frac{\tau^{\gamma}}{\left(\gamma+\mathbb{1}_{d}\right)^{m^{(\nu)}-\alpha}} .
$$


End of Proof of Theorem 2.1. Choose $M_{2}$ as

$$
M_{2} \geqslant \sum_{l, v=1}^{N} \sum_{0 \leqslant \alpha<m^{(v)}} C_{\alpha}^{(l, v)} e^{|m|} .
$$

Then, by (3.19), Proposition 2.2, Lemma 3.6 and (3.18)-0 we have



$$
\begin{aligned}
& \leqslant M_{1}\left\|\sum_{l, v=1}^{N} \sum_{0 \leqslant \alpha<m} \tau^{1_{d}} A_{\alpha}^{(l, v)}\left(z, \tau ; \partial_{z}\right)_{\Sigma} \mathscr{H}^{m^{(v)}-\alpha} v_{0, v}\right\|_{R^{\prime \prime}, s} \\
& \leqslant \sum_{l, v=1}^{N} \sum_{0 \leqslant \alpha<m^{(v)}} \frac{M_{1} C_{\alpha}^{(l, v)} \prod_{j=1}^{d} R_{j}^{\prime \prime}}{\left(s_{0}-s\right)^{\left|m^{(v) \mid}\right|-|\alpha|}}\left\|\mathscr{H}^{m^{(v)}-\alpha} v_{0, v}\right\|_{R^{\prime \prime}, s_{0}} \\
& =\sum_{l, v=1}^{N} \sum_{0 \leqslant \alpha<m^{(v)}} \frac{M_{1} C_{\alpha}^{(l, v)} \prod_{j=1}^{d} R_{j}^{\prime \prime}}{\left(s_{0}-s\right)^{|m|-|\alpha|}}\left\|\int_{[0,1]^{|m(v)|-|\alpha|}} v_{0, v}(z, \sigma \tau) d \sigma\right\|_{R^{\prime \prime}, s_{0}} \\
& \leqslant \sum_{l, v=1}^{N} \sum_{0 \leqslant \alpha<m^{(v)}} \frac{M_{1} C_{\alpha}^{(l, v)} \prod_{j=1}^{d} R_{j}^{\prime \prime}}{\left(s_{0}-s\right)^{|m|-|\alpha|}} \int_{[0,1]^{|m(v)|-|\alpha|}}\left\|v_{0, v}\right\|_{\sigma R^{\prime \prime}, s_{0}} d \sigma \\
& \leqslant \sum_{l, v=1}^{N} \sum_{0 \leqslant \alpha<m^{(v)}} \frac{M_{1}{ }^{2} C_{\alpha}^{(l, v)} \prod_{j=1}^{d} R_{j}^{\prime \prime}\|f\|_{R^{\prime}, s_{0}}}{\left(s_{0}-s\right)^{|m|}} \\
& \leqslant \frac{M_{1}^{2} M_{2} \prod_{j=1}^{d} R_{j}^{\prime \prime}\|f\|_{R^{\prime}, s_{0}}}{\left(s_{0}-s\right)^{|m|}} .
\end{aligned}
$$

Thus we have (3.18)-1. Now assume (3.18)- $p$ for a $p \in N$ and take arbitrary $s$ and $s^{\prime}$ with $0<s<s^{\prime}<s_{0}$. Then, by (3.19), Proposition 2.2 and Lemma 3.6 we have

$$
\begin{aligned}
& \left\|v_{p+1, \mu}\right\|_{R^{\prime \prime} . s}=\left\|\left(\partial_{\tau} \tau\right)^{m^{(\mu)}} \sum_{l, v=1}^{N} \sum_{0 \leqslant \alpha<m^{(v)}} \mathscr{H}_{Q+Q^{\prime}}^{(\mu, l)} \tau^{1^{d}} A_{\alpha}^{(l, v)}\left(z, \tau ; \partial_{z}\right)_{\Sigma} \mathscr{H}^{m^{(v)}-\alpha} v_{p, v}\right\|_{R^{\prime \prime}, s} \\
& \leqslant M_{1}\left\|\sum_{l, v=1}^{N} \sum_{0 \leqslant \alpha<m^{(v)}} \tau^{1_{d}} A_{\alpha}^{(l, v)}\left(z, \tau ; \partial_{z}\right)_{\Sigma} \mathscr{H}^{m^{(v)}-\alpha} v_{0, v}\right\|_{R^{\prime \prime}, s}
\end{aligned}
$$




$$
\begin{aligned}
& \leqslant \sum_{l, v=1}^{N} \sum_{0 \leqslant \alpha<m^{(v)}} \frac{M_{1} C_{\alpha}^{(l, v)} \prod_{j=1}^{d} R_{j}^{\prime \prime}}{\left(s^{\prime}-s\right)^{\left|m^{(v)}\right|-|\alpha|}}\left\|\mathscr{H}^{m^{(v)}-\alpha} v_{p, v}\right\|_{R^{\prime \prime}, s^{\prime}} \\
& \leqslant \sum_{l, v=1}^{N} \sum_{0 \leqslant \alpha<m^{(v)}} \frac{M_{1} C_{\alpha}^{(l, v)} \prod_{j=1}^{d} R_{j}^{\prime \prime}}{\left(s^{\prime}-s\right)^{\left|m^{(v)}\right|-|\alpha|}} \int_{[0,1]^{|m(v)|-|\alpha|}}\left\|v_{p, v}\right\|_{\sigma R^{\prime \prime}, s^{\prime}} d \sigma \\
& \leqslant \sum_{l, v=1}^{N} \sum_{0 \leqslant \alpha<m^{(v)}} \frac{M_{1}{ }^{p+2} M_{2}{ }^{p} C_{\alpha}^{(l, v)} \prod_{j=1}^{d} R_{j}^{\prime \prime}\|f\|_{R^{\prime}, s_{0}}}{\left(s^{\prime}-s\right)^{\mid m^{(v)|-| \alpha \mid}}} \\
& \times \int_{[0,1]|m(v)|-|\alpha|} \frac{\left(\prod_{j=1}^{d}\left(\prod_{i=1}^{m_{j}^{(v)}-\alpha_{j}} \sigma_{j, i} R_{j}^{\prime \prime}\right)\right)^{p}}{\left(s_{0}-s^{\prime}\right)^{|m| p}} d \sigma \\
& =\sum_{l, v=1}^{N} \sum_{0 \leqslant \alpha<m^{(v)}} \frac{C_{\alpha}^{(l, v)} M_{1}{ }^{p+2} M_{2}{ }^{p}\left(\prod_{j=1}^{d} R_{j}^{\prime \prime}\right)^{p+1}\|f\|_{R^{\prime}, s_{0}}}{\left(s_{0}-s^{\prime}\right)^{|m| p}\left(s^{\prime}-s\right)^{\left|m^{(v) \mid}\right|-|\alpha|}(p+1)^{\left|m^{(v)}\right|-|\alpha|}} .
\end{aligned}
$$

Hence, choosing $s^{\prime}-s=\left(s_{0}-s\right) /(p+1)$ we have

$$
\begin{aligned}
\left\|v_{p+1, \mu}\right\|_{R^{\prime \prime}, s} & \leqslant \sum_{l, v=1}^{N} \sum_{0 \leqslant \alpha<m^{(v)}} \frac{C_{\alpha}^{(l, v)} M_{1}{ }^{p+2} M_{2}{ }^{p}\left(\prod_{j=1}^{d} R_{j}^{\prime \prime}\right)^{p+1}\|f\|_{R^{\prime}, s_{0}}}{\left(\frac{p+1}{p}\right)^{|m| p}} \\
& \leqslant \sum_{l, v=1}^{N} \sum_{0 \leqslant \alpha^{\prime}<m^{(v)}} \frac{C_{\alpha}^{(l, v)} M_{1}{ }^{p+2} M_{2}{ }^{p} e^{|m|}\left(\prod_{j=1}^{d} R_{j}^{\prime \prime}\right)^{p+1}\|f\|_{R^{\prime}, s_{0}}}{\left(s_{0}-s\right)^{|m|(p+1)}} \\
& \leqslant \frac{M_{1}{ }^{p+2} M_{2}{ }^{p+1}\left(\prod_{j=1}^{d} R_{j}^{\prime \prime}\right)^{p+1}\|f\|_{R^{\prime}, s_{0}}}{\left(s_{0}-s\right)^{|m|(p+1)}}
\end{aligned}
$$

Hence we can prove (3.18)- $(p+1)$. Set $\delta:=1 /\left(M_{1} M_{2}\right)$ and take an arbitrary vector $R^{\prime \prime}$ which satisfies $0 \prec R^{\prime \prime} \leqslant R^{\prime}$ and $\prod_{j=1}^{d} R_{j}^{\prime \prime}<\delta\left(s_{0}-s\right)^{|m|}$. Then it follows that 


$$
\begin{aligned}
\sum_{p=0}^{\infty}\left\|v_{p}\right\|_{R^{\prime \prime}, s} & \leqslant M_{1}\|f\|_{R^{\prime}, s_{0}} \sum_{p=0}^{\infty}\left(\frac{M_{1} M_{2} \prod_{j=1}^{d} R_{j}^{\prime \prime}}{\left(s_{0}-s\right)^{|m|}}\right)^{p} \\
& <\infty
\end{aligned}
$$

Thus there exists $v(z, \tau) \in E\left(B\left(R^{\prime \prime}\right) ; X_{s}\right)^{\oplus N}$ such that $\sum_{j=0}^{p} v_{j}$ converges to $v$ as $p$ tends to infinity in $E\left(B\left(R^{\prime \prime}\right) ; X_{s}\right)^{\oplus N}$. Set

$$
u:=\left(\begin{array}{rrrr}
\mathscr{H}^{m^{(1)}} & & 0 & \\
& \ddots & & v . \\
0 & & \mathscr{H}^{m^{(N)}} &
\end{array}\right)
$$

Then it is obvious that $P_{\Sigma} u=f$ holds and $u$ is holomorphic on

$$
\bigcup_{0<s<s_{0}}\left(\Omega_{s} \times\left\{\tau \in B\left(R^{\prime}\right) ; \prod_{j=1}^{d}\left|\tau_{j}\right|<\delta\left(s_{0}-s\right)^{|m|}\right\}\right) .
$$

Hence the existence is proved. Next we will show the uniqueness. Assume that $u(z, \tau) \in E\left(B\left(R^{\prime \prime}\right) ; X_{s}\right)^{\oplus N}$ satisfies $P_{\Sigma} u=0$. Then, by the preceding argument for some positive constant $C$ we have

$$
\|u\|_{R^{\prime \prime}, s} \leqslant C\left(\frac{M_{1} M_{2} \prod_{j=1}^{d} R_{j}^{\prime \prime}}{\left(s_{0}-s\right)^{|m|}}\right)^{p}
$$

for any $0<s<s_{0}<1$ and $p \in N$. Therefore, choosing $M_{1} M_{2} \prod_{j=1}^{d} R_{j}^{\prime \prime}<\left(s_{0}-s\right)^{|m|}$ and letting $p$ tend to infinity, we see that $u \equiv 0$ in some neighborhood of $(z, \tau)=\left(z_{0}, 0\right)$. Thus the uniqueness follows. Therefore the proof is complete.

\section{§4. Applications}

Let $M$ be $R_{x}^{n} \times R_{t}^{d}$ with its complexification $X:=C_{z}^{n} \times C_{\tau}^{d}=Y \times C^{d}$ and $\pi_{M}$ the canonical projection $T_{M}^{*} X \rightarrow M$. Set $N:=R^{n} \cong M \cap\{t=0\} \hookrightarrow M, L:=X$ $\cap\{\operatorname{Im} z=0\}=R^{n} \times C^{d}, \tilde{\Lambda}:=T_{L}^{*} X \cong T_{N}^{*} Y \times C^{d}$ and $\Lambda:=T_{M}^{*} X \cap \tilde{\Lambda}$. We denote the sheaf of microfunctions on $T_{M}^{*} X$ (resp. $T_{N}^{*} Y$ ) by $\mathscr{C}_{M}$ (resp. $\mathscr{C}_{N}$ ) as usual. Further, let $\mathscr{C O}{ }_{L}$ be the sheaf of microfunctions with holomorphic parameters on $\tilde{\Lambda}$; that is, 


$$
\mathscr{C} \mathcal{O}_{L}:=\mu_{L}\left(\mathcal{O}_{X}\right) \otimes o r_{N / Y}[n]
$$

where $\mu_{L}$ denotes Sato's microlocalization functor along $L$ and $o r_{N / Y}$ denotes the relative orientation sheaf (see $[\mathrm{K}-\mathrm{Sc} 2]$ and $[\mathrm{S}-\mathrm{K}-\mathrm{K}]$ ). The sheaf $\mathscr{B}_{M}$ of hyperfunctions on $M$ and the sheaf $\mathscr{B} C_{L}$ of hyperfunctions with holomorphic parameters on $L$ are defined by $\mathscr{B}_{M}:=\left.\mathscr{C}_{M}\right|_{M}$ and $\mathscr{B} \mathcal{O}_{L}:=\left.\mathscr{C O}_{L}\right|_{L}$ respectively. Let $\rho$ be a natural mapping $N \underset{M}{\times} T_{M}^{*} X \ni(x, 0 ; \sqrt{-1}(\langle\xi, d x\rangle+\langle\eta, d t\rangle)) \mapsto(x ; \sqrt{-1}<\xi$, $d x\rangle) \in T_{N}^{*} Y$. Then, we have the following canonical morphisms:

$$
\left.\left.\mathscr{C} \mathcal{O}_{L}\right|_{\Lambda} \longrightarrow \mathscr{C}_{M}\right|_{\Lambda}, \quad \rho_{!}\left(\left.\mathscr{C}_{M}\right|_{N_{M} T_{M}^{*} X}\right) \rightarrow \mathscr{C}_{N}
$$

Set $p_{0}:=\left(0 ; \sqrt{-1} d x_{1}\right) \in T_{N}^{*} Y$ and assume that $P\left(x, t ; \partial_{x}, \partial_{t}\right)$ is a matrix of microdifferential operators of Fuchsian type with weight $(k, m)$ defined in some neighborhood of $\rho^{-1}\left(p_{0}\right)$, then the following morphism is induced:

$$
P: \rho_{!}\left(\left.\mathscr{C}_{M}\right|_{N_{M} \times T_{M}^{*} X}\right)_{p_{0}} \rightarrow \rho_{!}\left(\left.\mathscr{C}_{M}\right|_{N_{M} \times T_{M}^{*} X}\right)_{p_{0}},
$$

where $\rho_{!}\left(\left.\mathscr{C}_{M}\right|_{N_{M} \times T_{M}^{*} X}\right)_{p_{0}}$ denotes the stalk at $p_{0}$.

Consider the following condition:

【A-2】。 $\operatorname{det}\left(\sigma_{\left|m^{(v) \mid}\right|}\left(P^{(\mu, v)}\right)(z, \tau ; \zeta, \eta)\right)=\tau^{\tilde{k}} \widetilde{P}(z, \tau ; \zeta, \eta)$ for a function $\widetilde{P}\left(\tilde{k}:=\sum_{v=1}^{N} k^{(v)}\right.$ $\in \mathbb{N}_{0}{ }^{d}$ ) which satisfies the following condition:

There exist positive constants $h_{0}, M$ and $v_{i}$ with $v_{i} \geqslant 1(1 \leqslant i \leqslant d)$ such that $\tilde{P}(z, t ; \zeta, \eta)$ never vanishes on the set

$$
\begin{aligned}
& \left\{(z, t ; \zeta, \eta) \in \mathbb{C}^{n} \times \mathbb{R}^{d} \times \mathbb{C}^{n} \times \mathbb{C}^{d} ;|z|,|t|<h_{0},\left|\zeta_{j}\right|<h_{0}\left|\zeta_{1}\right|(2 \leqslant j \leqslant n),\right. \\
& \left.\quad \exists \lambda>M\left(\sum_{j=1}^{n}\left|\operatorname{Im} z_{j}\right|+\sum_{j=2}^{n}\left|\operatorname{Im}\left(\zeta_{j} / \zeta_{1}\right)\right|\right),\left|\operatorname{Im}\left(\eta_{i} / \zeta_{1}\right)\right|=v_{i} \lambda(1 \leqslant i \leqslant d)\right\} .
\end{aligned}
$$

4.1. Example. (1) Let $P$ be as in Example 1.5 (2) with $d=2$. Set

$$
\left\{\begin{array}{l}
b_{i j}(z, \tau ; \zeta):=\sigma_{1}\left(B_{i j}\right)(z, \tau ; \zeta) \\
d_{i j}(z, \tau ; \zeta):=\sigma_{2}\left(D_{i j}\right)(z, \tau ; \zeta)
\end{array}\right.
$$

and suppose that $b_{21}(z, \tau ; \zeta)=d_{21}(z, \tau ; \zeta)=0$. Then

$$
\begin{aligned}
& \operatorname{det}\left(\sigma_{2}\left(P^{(i, j)}\right)(z, \tau ; \zeta, \eta)\right) \\
& \quad=\tau_{1} \tau_{2} \operatorname{det}\left(\begin{array}{cc}
\eta_{1}{ }^{2}+\tau_{2}\left(b_{11} \eta_{1}+d_{11}\right) & \tau_{1} b_{12} \eta_{2}+d_{12} \\
0 & \eta_{2}{ }^{2}+\tau_{1}\left(b_{22} \eta_{2}+d_{22}\right)
\end{array}\right)
\end{aligned}
$$




$$
=\tau_{1} \tau_{2}\left(\eta_{1}^{2}+\tau_{2}\left(b_{11} \eta_{1}+d_{11}\right)\right)\left(\eta_{2}^{2}+\tau_{1}\left(b_{22} \eta_{2}+d_{22}\right)\right) \text {. }
$$

Hence if $\eta_{1}{ }^{2}+\tau_{2}\left(b_{11}(z, \tau ; \zeta) \eta_{1}+d_{11}(z, \tau ; \zeta)\right)$ (resp. $\eta_{2}{ }^{2}+\tau_{1}\left(b_{22}(z, \tau ; \zeta) \eta_{2}+d_{22}(z\right.$, $\tau ; \zeta))$ ) is hyperbolic with respect to $\eta_{1}$ (resp. $\eta_{2}$ ), then we see that [A-2] is satisfied (cf. Kashiwara-Kawai $[\mathrm{K}-\mathrm{K}]$ ).

(2) Condition $[\mathrm{A}-2]$ is satisfied if

$$
\tilde{P}(x, t ; \xi, \eta)=\prod_{i=1}^{d} P_{i}\left(x, t ; \xi, \eta_{1}, \ldots, \eta_{i}\right),
$$

where each $P_{i}$ satisfies the following condition: there exist positive constants $h_{0}$ and $M_{i}(1 \leqslant i \leqslant d)$

such that $P_{i}\left(z, t ; \zeta, \eta_{1}, \cdots, \eta_{i}\right)$ never vanishes on the set

$$
\begin{array}{r}
\left\{\left(z, t ; \zeta, \eta_{1}, \ldots, \eta_{i}\right) \in C^{n} \times \mathbb{R}^{d} \times C^{n+i} ;|z|,|t|<h_{0},\left|\zeta_{j}\right|<h_{0}\left|\zeta_{1}\right|(2 \leqslant j \leqslant n),\right. \\
\left.\left|\operatorname{Im}\left(\eta_{i} / \zeta_{1}\right)\right|>M_{i}\left(\sum_{j=1}^{n}\left|\operatorname{Im} z_{j}\right|+\sum_{j=2}^{n}\left|\operatorname{Im}\left(\zeta_{j} / \zeta_{1}\right)\right|+\sum_{j=1}^{i-1}\left|\operatorname{Im}\left(\eta_{j} / \zeta_{1}\right)\right|\right)\right\} .
\end{array}
$$

4.2. Theorem. Assume that P satisfies [A-1] and [A-2]. Then, for any microfunctions with holomorphic parameters

$$
f(x, t), g(x, t) \in \rho_{*}\left(\left.\mathscr{C} \mathcal{C}_{L}\right|_{N_{M} T_{M}^{*}}\right)_{p_{0}}^{\oplus N},
$$

there exists an $\mathrm{N}$-tuple of microfunctions

$$
u(x, t) \in \rho_{!}\left(\left.\mathscr{C}_{M}\right|_{N_{M} \times T_{M}^{*} X}\right)_{p_{0}}^{\oplus N}
$$

such that $u$ is a solution of the Goursat problem

$$
\left\{\begin{aligned}
P\left(x, t ; \partial_{x}, \partial_{t}\right) u(x, t) & =f(x, t), \\
u(x, t)-g(x, t) & =O\left(t^{m-k}\right) .
\end{aligned}\right.
$$

4.3. Remark. The uniqueness of $u(x, t)$ in Theorem 4.2 will be discussed in a forthcoming paper (cf. [O3] and [O4]).

Proof of Theorem 4.2. We may assume that $g \equiv 0$. We remark that the 
method of proof is similar to Oaku [O2].

Step 1. Set

$$
W:=\left\{(z ; \zeta) \in C^{2 n} ;|z|<h_{0},\left|\zeta_{j}\right|<h_{0}\left|\zeta_{1}\right|(2 \leqslant j \leqslant n)\right\}
$$

and choose constants $h$ and $c$ such that

$$
0<h<\min \left\{h_{0} /\left(4 n^{2}\right), 1 / 2\right\}, 0<c<\min \left\{r_{0}, h_{0}\right\}
$$

where $r_{0}>0$ is a constant determined by $P$ and $W$ by Theorem 1.3. Then, setting

$$
\left\{\begin{array}{l}
\Sigma:=\left\{z \in \mathbb{C}^{n} ; z_{1}=\sqrt{-1} c\right\}, \\
\Omega^{\prime}:=\left\{z \in \mathbb{C}^{n} ;\left|z_{j}\right|+\left|z_{1}-\sqrt{-1} c\right| / h_{0}<c / h(2 \leqslant j \leqslant n)\right\},
\end{array}\right.
$$

we see that $\Omega^{\prime}$ is $h$ - $\Sigma$-flat, dia $\Omega^{\prime}<r_{0}$ and $\Omega^{\prime} \subset\left\{z \in C^{n} ;|z|<h\right\}$. Choose constants $a$ and $a_{1}$ such that

$$
0<a<a_{1}<\left(2 n^{2}-1\right) c
$$

Then we have $\left\{z \in C^{n} ;|z|<a_{1}\right\} \subset \Omega^{\prime}$ by (4.5) and (4.6). On the other hand, by Theorem 2.2 of Kataoka-Tose [Kt-To] and the argument to the proof of Theorem 3.8.1 of Kashiwara-Kawai-Kimura $\left[\mathrm{K}^{3}\right]$, we can find holomorphic functions $F(z, \tau)={ }^{t}\left(F_{1}(z, \tau), \ldots, F_{N}(z, \tau)\right)$ defined in a complex neighborhood of

$$
\begin{gathered}
\left\{(z, \tau) \in \mathbb{C}^{n+d} ;|\operatorname{Re} z|,|\operatorname{Re} \tau|<h_{1},|\operatorname{Im} \tau|<h_{2}, h \sum_{j=2}^{n}\left|\operatorname{Im} z_{j}\right|<2(n-1) \operatorname{Im} z_{1}<h^{\prime}\right\} \\
\cup\left\{(x, t) \in \mathbb{R}^{n+d} ; a \leqslant|x|<h_{1}, t \mid<h_{1}\right\}
\end{gathered}
$$

with some constants $h^{\prime}, h_{2}>0$ and $0<h_{0}<h_{1}$ such that on $\rho^{-1}\left(p_{0}\right)$

$$
\left(\begin{array}{c}
\operatorname{sp}_{M}\left(\mathrm{~b}\left(F_{1}\right)\right) \\
\vdots \\
\operatorname{sp}_{M}\left(\mathrm{~b}\left(F_{N}\right)\right)
\end{array}\right)=f
$$

Here $\operatorname{sp}_{M}: \pi_{M}^{-1} \mathscr{B}_{M} \rightarrow \mathscr{C}_{M}$ denotes the spectral morphism from the sheaf of hyperfunctions to that of microfunctions and $\mathrm{b}\left(F_{v}\right)$ denotes the boundary value as a hyperfunction of $F_{v}$. Set 


$$
\begin{aligned}
\tilde{D} & :=\left\{(x, t) \in \boldsymbol{R}^{n+d} ;|x|<h_{1},|t|<h_{1}\right\}, \\
\tilde{G} & :=\left\{(y, s) \in \boldsymbol{R}^{n+d} ;|s|<h_{2}, h \sum_{j=2}^{n}\left|y_{j}\right|<(n-1) y_{1}\right\}, \\
\tilde{G}^{\prime} & :=\left\{(y, s) \in \boldsymbol{R}^{n+d} ;|s|<h_{2}, h \sum_{j=2}^{n}\left|y_{j}\right|<2(n-1) y_{1}\right\}, \\
\tilde{G}_{h^{\prime}} & :=\left\{(y, s) \in \boldsymbol{R}^{n+d} ;|s|<h_{2}, h \sum_{j=2}^{n}\left|y_{j}\right|<(n-1)\left(y_{1}+h^{\prime}\right)\right\} .
\end{aligned}
$$

Then, we have

$$
\begin{aligned}
& \tilde{G}_{h^{\prime}}=\left(\tilde{G}_{h^{\prime}} \cap\left\{(y, s) \in \boldsymbol{R}^{n+d} ; y_{1}<h^{\prime}\right\}\right) \cup\left(\tilde{G}_{h^{\prime}} \cap \widetilde{G}^{\prime}\right), \\
& F \in \mathcal{O}\left(\tilde{D}+\sqrt{-1}\left(\tilde{G}^{\prime} \cap\left\{(y, s) \in \boldsymbol{R}^{n+d} ; y_{1}<h^{\prime}\right\}\right)\right)^{\oplus N} .
\end{aligned}
$$

Therefore, since $\tilde{D}+\sqrt{-1} \widetilde{G}_{h^{\prime}}$ is a Stein open set we can decompose $F$ into

$$
F=\tilde{f}+f^{\prime}
$$

with

$$
\left\{\begin{array}{l}
\tilde{f} \in \mathcal{O}\left(\tilde{D}+\sqrt{-1}\left(\tilde{G}_{h^{\prime}} \cap \tilde{G}^{\prime}\right)\right)^{\oplus N}, \\
f^{\prime} \in \mathcal{O}\left(\tilde{D}+\sqrt{-1}\left(\tilde{G}_{h^{\prime}} \cap\left\{(y, s) \in \boldsymbol{R}^{n+d} ; y_{1}<h^{\prime}\right\}\right)\right)^{\oplus N} .
\end{array}\right.
$$

Since an $N$-tuple $f^{\prime}$ is holomorphic in a complex neighborhood of $\tilde{D}$ by (4.7), (4.8) and (4.9), we see that an $N$-tuple $\tilde{f}$ is holomorphic in some complex neighborhood of

$$
(\tilde{D}+\sqrt{-1} \tilde{G}) \cup\left\{(x, t) \in \boldsymbol{R}^{n+d} ; a \leqslant|x|<h_{1},|t|<h_{1}\right\},
$$

and on $\rho^{-1}\left(p_{0}\right)$

$$
\left(\begin{array}{c}
\operatorname{sp}_{M}\left(\mathrm{~b}\left(\tilde{f}_{1}\right)\right) \\
\vdots \\
\operatorname{sp}_{M}\left(\mathrm{~b}\left(\tilde{f}_{N}\right)\right)
\end{array}\right)=f
$$

Step 2. Set 


$$
\left\{\begin{array}{l}
D:=\left\{x \in \mathbb{R}^{n} ;|x|<h_{1}\right\} \\
G:=\left\{y \in \mathbb{R}^{n} ; h \sum_{j=2}^{n}\left|y_{j}\right|<(n-1) y_{1}\right\} . \\
\Omega:=\Omega^{\prime} \cap\left(\mathbb{R}^{n}+\sqrt{-1} G\right)=\Omega^{\prime} \cap(D+\sqrt{-1} G) .
\end{array}\right.
$$

Then, by (4.10) and (4.11) we see that $\Omega$ is $h_{0}-\Sigma$-flat, dia $\Omega<r_{0}$ and an $N$-tuple $\tilde{f}$ is holomorphic on

$$
\Omega \times B\left(h_{2} \mathbb{1}_{d}\right)
$$

(recall that $\left.\mathbb{1}_{d}:=(1, \ldots, 1) \in N^{d}\right)$. Consider the following:

$$
\left\{\begin{aligned}
P_{\Sigma} \tilde{u} & =\tilde{f} \\
\tilde{u} & =O\left(\tau^{m-k}\right)
\end{aligned}\right.
$$

Thus, by Theorem 1.3 , there exists a constant $\delta$ with $0<\delta<1$ such that there exist a unique holomorphic solution $\tilde{u}$ on

$$
\bigcup_{0<s<1}\left(\Omega_{s} \times\left\{\tau \in B\left(h_{2} \mathbb{1}_{d}\right) ; \prod_{j=1}^{d}\left|\tau_{j}\right|<\delta(1-s)^{|m|}\right\}\right),
$$

where

$$
\Omega_{s}:=\left\{s z+(1-s) \sqrt{-1}(c, 0, \ldots, 0) \in C^{n} ; z \in \Omega\right\}
$$

Step 3. Take any $x_{0}$ with $a \leqslant\left|x_{0}\right| \leqslant a_{1}$. Note that $\tilde{f}(z, \tau)$ is holomorphic at $(z, \tau)=\left(x_{0}, 0\right)$. Hence we can choose sufficiently small constants $c^{\prime}$ and $T_{1}$ with $0<c^{\prime}<c$ such that

$$
\Omega^{\prime \prime}:=\left\{z \in \mathbb{C}^{n} ; 2\left|z_{j}-x_{0, j}\right|+\frac{2}{h_{0}}\left|z_{1}-x_{0,1}-\sqrt{-1} c^{\prime}\right|<\frac{c^{\prime}}{h}\right\} \Subset \Omega^{\prime}
$$

and

$\tilde{f}$ is holomorphic on $\Omega^{\prime \prime} \times\left\{\tau \in C^{d} ;\left|\tau_{j}\right|<T_{1}(1 \leqslant j \leqslant d)\right\}$.

Set

$$
\Sigma^{\prime}:=\left\{z \in \mathbb{C}^{n} ; z_{1}=x_{0.1}+\sqrt{-1} c^{\prime}\right\}
$$

then we see that $\Omega^{\prime \prime}$ is $h_{0}-\Sigma$-flat and $\Sigma^{\prime} \cap \Omega^{\prime \prime} \subset \Omega_{s_{1}}$ for some $0<s_{1}<1$. Therefore, by the argument to Théorème 2.5.1 of Bony-Schapira [Bo-Sc] $\mathbb{P}_{\Sigma} \tilde{u}-P_{\Sigma^{\prime}} \tilde{u}$ is holomorphic on $\Omega^{\prime \prime} \times B\left(\delta\left(1-s_{1}\right)^{|m|} \mathbb{1}_{d}\right)$. Since $P_{\Sigma} \tilde{u}=\tilde{f}$ is holomorphic on 
$\Omega^{\prime \prime} \times B\left(T_{1} \mathbf{1}_{d}\right), P_{\Sigma^{\prime}} \tilde{u}$ is holomorphic on $\Omega^{\prime \prime} \times B\left(\min \left\{T_{1}, \delta\left(1-s_{0}\right)^{|m|} \mathbb{1}_{d}\right\}\right)$. Thus by Theorem 1.3, there exists an $N$-tuple $\tilde{u}^{\prime}$ of holomorphic functions on a neighborhood of $\Omega^{\prime} \times\{0\}$ such that

$$
\left\{\begin{aligned}
P_{\Sigma^{\prime}} \tilde{u}^{\prime} & =P_{\Sigma^{\prime}} \tilde{u} \\
\tilde{u}^{\prime} & =O\left(\tau^{m-k}\right) .
\end{aligned}\right.
$$

By the uniqueness of $\tilde{u}^{\prime}$, we have $\tilde{u}^{\prime}=\tilde{u}$ on a neighborhood of $\left(\Omega \cap \Omega^{\prime \prime}\right) \times\{0\}$ because $x_{0} \in \Omega^{\prime \prime}$. Since $\left\{x \in \mathbb{R}^{n} ; a \leqslant|x| \leqslant a_{1}\right\}$ is compact, it follows that $\tilde{u}$ is analytically continued to some neighborhood of

$$
\begin{aligned}
U_{1}:= & \bigcup_{0<s<1}\left(\Omega_{s} \times\left\{\tau \in B\left(h_{2} \mathbb{1}_{d}\right) ; \prod_{j=1}^{d}\left|\tau_{j}\right|<\delta(1-s)^{|m|}\right\}\right) \\
& \cup\left\{(z, \tau) \in C^{n+d} ; a \leqslant|\operatorname{Re} z| \leqslant a_{1},|\operatorname{Im} z|<a^{\prime},|\tau|<T_{2}\right\}
\end{aligned}
$$

with some $T_{2}$ and $a^{\prime}>0$.

Step 4. Fix arbitrary $\sigma=\left(\sigma_{1}, \ldots, \sigma_{d}\right) \in\{-1,1\}^{d}$. For simplicity, set

$$
D^{\prime}:=\left\{(z, t) \in \boldsymbol{C}^{n} \times \boldsymbol{R}^{d} ;|\operatorname{Re} z|<a_{1},|\operatorname{Im} z|<a^{\prime}\right\} \subset \boldsymbol{C}^{n} \times \boldsymbol{R}^{d} .
$$

Then, by the result of Step 3 we see that there exists a positive constant $\delta_{1}$ such that $\tilde{u}$ is holomorphic in some complex neighborhood of

$$
\begin{aligned}
& \left\{(z, t) \in D^{\prime} ; \sum_{j=1}^{d}\left|\sigma_{j} v_{j} t_{j}\right|<T_{2}, \operatorname{Im} z_{1}>\delta_{1}\left(\sum_{j=1}^{d}\left|\sigma_{j} v_{j} t_{j}\right|\right)^{1 /|m|}+\frac{h}{n-1} \sum_{j=2}^{n}\left|\operatorname{Im} z_{j}\right|\right\} \\
& \cup\left\{(z, t) \in D^{\prime} ; \sum_{j=1}^{d}\left|\sigma_{j} v_{j} t_{j}\right|<T_{2}, \operatorname{Im} z_{1}>\delta_{1}\left(\prod_{j=1}^{d}\left|\sigma_{j} v_{j} t_{j}\right|\right)^{1 /|m|}+\frac{h}{n-1} \sum_{j=2}^{n}\left|\operatorname{Im} z_{j}\right|\right\} \\
& \cup\left\{(z, \tau) \in C^{n+d} ; a \leqslant|\operatorname{Re} z| \leqslant a_{1},|\operatorname{Im} z|<a^{\prime}, \sum_{j=1}^{d}\left|\sigma_{j} v_{j} t_{j}\right|<T_{2}\right\} .
\end{aligned}
$$

Choose positive constants $M_{1}$ and $T_{0}$ such that

$$
\left\{\begin{array}{r}
\max \left\{|m| n M, \delta_{1} / a^{\prime}\right\}<M_{1}, \\
0<T_{0}<\min \left\{T_{2}, 1\right\}, \\
\exp \left(M_{1} T_{0}\right)<2, \\
2 n \exp \left(M_{1} T_{0}\right) M_{1} T_{0}{ }^{1 /|m|}<h_{0}, \\
\delta_{1} T_{0}{ }^{1 /|m|}<\frac{a^{\prime}}{2},
\end{array}\right.
$$


and set

$$
\varphi_{2, \varepsilon}^{\sigma}\left(y^{\prime}, t\right):=\left(M_{1}\left(\sum_{j=1}^{d} \sigma_{j} v_{j} t_{j}\right)^{1 /|m|}+n h\right) \sqrt{\left|y^{\prime}\right|^{2}+\varepsilon^{2}}
$$

where $y^{\prime}:=\left(y_{2}, \ldots, y_{n}\right)$ and $\varepsilon>0$. Let $\varphi_{1}(T)$ be a real valued real analytic function in a neighborhood of $\left\{T \in R ; 0<T \leqslant T_{0}\right\}$ satisfying for some $h^{\prime \prime}>0$

$$
\begin{cases}\varphi_{1}\left(T_{0}\right)>\delta_{1} T_{0}{ }^{1 /|m|}, & \text { for any } 0<T \leqslant T_{0} \\ \varphi_{1}(T) \geqslant h^{\prime \prime} & \text { for any } 0<T \leqslant T_{0} \\ \left(\frac{d \varphi_{1}}{d T}\right)(T)>0 & \end{cases}
$$

Hence there exists a small constant $c_{1}$ such that if $0<T<c_{1}$ or $T_{0}-c_{1}<T<T_{0}$, then

$$
\varphi_{1}(T)>\delta_{1} T^{1 /|m|}
$$

Set

$$
\begin{gathered}
\varphi_{1, \varepsilon}^{\sigma}(t):=\varphi_{1}\left(\sum_{j=1}^{d} \sigma_{j} v_{j} t_{j}\right) \\
\varphi_{\varepsilon}^{\sigma}\left(y^{\prime}, t\right):=\left(\varphi_{1, \varepsilon}^{\sigma}(t)+\varphi_{2, \varepsilon}^{\sigma}\left(y^{\prime}, t\right)\right) \exp \left(M_{1} \sum_{j=1}^{d} \sigma_{j} v_{j} t_{j}\right) .
\end{gathered}
$$

Then we see easily see that

$$
\frac{\partial \varphi_{\varepsilon}^{\sigma}}{\partial t_{j}}\left(y^{\prime}, t\right)=\sigma_{j} v_{j} \lambda \quad(1 \leqslant j \leqslant d)
$$

where

$$
\begin{aligned}
\lambda:= & M_{1} \varphi_{\varepsilon}^{\sigma}\left(y^{\prime}, t\right)+\exp \left(M_{1} \sum_{j=1}^{d} \sigma_{j} v_{j} t_{j}\right) \\
& \times\left(\frac{d \varphi_{1}}{d T}\left(\sum_{j=1}^{d} \sigma_{j} v_{j} t_{j}\right)+\frac{M_{1}}{|m|}\left(\sum_{j=1}^{d} \sigma_{j} v_{j} t_{j}\right)^{1 /|m|-1} \sqrt{\left|y^{\prime}\right|^{2}+\varepsilon^{2}}\right) .
\end{aligned}
$$

Moreover by (4.4) and (4.14) we have 


$$
\begin{aligned}
\lambda>M_{1}\left(\varphi_{\varepsilon}^{\sigma}\left(y^{\prime}, t\right)+\frac{M_{1}}{|m|}\left(\sum_{j=1}^{d} \sigma_{j} v_{j} t_{j}\right)^{1 /|m|-1}\left|y^{\prime}\right|\right) \\
>M\left(\varphi_{\varepsilon}^{\sigma}\left(y^{\prime}, t\right)+\sum_{j=2}^{n}\left|y_{j}\right|\right)
\end{aligned}
$$

and

$$
\sum_{j=2}^{n}\left|\frac{\partial \varphi_{\varepsilon}^{\sigma}}{\partial z_{j}}\right|=\sum_{j=2}^{n} \frac{1}{2}\left|\frac{\partial \varphi_{\varepsilon}^{\sigma}}{\partial y_{j}}\right|<\frac{n-1}{2}\left(M_{1} T_{0}{ }^{1 /|m|}+n h\right) \exp \left(M_{1} T_{0}\right)<\frac{h_{0}}{2}
$$

By (4.10), $\tilde{f}$ is holomorphic in complex neighborhood of

$$
\left\{(z, t) \in D^{\prime} ; 0 \leqslant \sigma_{j} v_{j} t_{j}(1 \leqslant j \leqslant d), \sum_{j=1}^{d} \sigma_{j} v_{j} t_{j}<T_{0}, \operatorname{Im} z_{1}>\frac{h}{n-1} \sum_{j=2}^{n}\left|\operatorname{Im} z_{j}\right|\right\}
$$

4.4. Lemma. Assume that $\tilde{u}$ is holomorphic in a complex neighborhood of

$$
\left\{(z, t) \in D^{\prime} ; 0<\sigma_{j} v_{j} t_{j}(1 \leqslant j \leqslant d), 0<\sum_{j=1}^{d} \sigma_{j} v_{j} t_{j}<T_{0}, \operatorname{Im} z_{1}>\varphi_{\varepsilon}^{\sigma}\left(\operatorname{Im} z^{\prime}, t\right)\right\} .
$$

Then $\tilde{u}$ is analytically continued to some complexification of

$$
\left\{(z, t) \in D^{\prime} ; 0<\sigma_{j} v_{j} t_{j}(1 \leqslant j \leqslant d), 0<\sum_{j=1}^{d} \sigma_{j} v_{j} t_{j}<T_{0}, \operatorname{Im} z_{1}=\varphi_{\varepsilon}^{\sigma}\left(\operatorname{Im} z^{\prime}, t\right)\right\} .
$$

Proof of Lemma 4.4. We can apply the argument to Lemma 4.3 of Kashiwara-Kawai [K-K]. Set

$$
V:=\left\{(z, t) \in D^{\prime} ; 0<\sigma_{j} v_{j} t_{j}(1 \leqslant j \leqslant d), 0<\sum_{j=1}^{d} \sigma_{j} v_{j} t_{j}<T_{0}, \operatorname{Im} z_{1}=\varphi_{\varepsilon}^{\sigma}\left(\operatorname{Im} z^{\prime}, t\right)\right\} .
$$

We regard $V$ as a real analytic manifold of dimension $2 n+d-1$ with coordinates $x:=\operatorname{Re} z, y:=\operatorname{Im} z$ and $t$. Let $\tilde{V}$ be a complex neighborhood of $V$ and set $\widetilde{\mathscr{A}_{V}}:=H^{0}\left(v_{V}\left(\mathcal{O}_{\tilde{V}}\right)\right)$, where $v_{V}(*)$ denotes the specialization functor along $V$ (see $[\mathrm{K}-\mathrm{Sc} 2]$ and $[\mathrm{S}-\mathrm{K}-\mathrm{K}]$ ). Since $\tilde{u}$ is analytic on

$$
\left\{(z, t) \in D^{\prime} ; 0<\sigma_{j} v_{j} t_{j}(1 \leqslant j \leqslant d), 0<\sum_{j=1}^{d} \sigma_{j} v_{j} t_{j}<T_{0}, \operatorname{Im} z_{1}>\varphi_{\varepsilon}^{\sigma}\left(\operatorname{Im} z^{\prime}, t\right)\right\},
$$


it follows that $\tilde{u}$ defines a section of $\mathscr{A}_{V}{ }^{\oplus N}$ on the set

$$
\begin{gathered}
\left\{(x, y, t)+\sqrt{-1}\left(\left(c+2 \operatorname{Re}\left(\left\langle\zeta^{\prime}, \frac{\partial \varphi_{\varepsilon}^{\sigma}}{\partial z^{\prime}}\right\rangle\right)\right) \frac{\partial}{\partial x_{1}}+2 \operatorname{Im}\left(\left\langle\zeta^{\prime}, \frac{\partial \varphi_{\varepsilon}^{\sigma}}{\partial z^{\prime}}\right\rangle\right) \frac{\partial}{\partial y_{1}}\right.\right. \\
\left.\left.+\left\langle\operatorname{Im} \zeta^{\prime}, \frac{\partial}{\partial x^{\prime}}\right\rangle-\left\langle\operatorname{Re} \zeta^{\prime}, \frac{\partial}{\partial y^{\prime}}\right\rangle\right) \in \mathbb{T}_{V} \tilde{V} ; \zeta^{\prime} \in \mathbb{C}^{n-1}, c>0\right\} \\
=\left\{(x, y, t)+\left(\sqrt{-1} c \frac{\partial}{\partial z_{1}}+\sqrt{-1} c \frac{\partial}{\partial \bar{z}_{1}}+\left\langle\zeta^{\prime}, \frac{\partial}{\partial z^{\prime}}+2 \sqrt{-1} \frac{\partial \varphi_{\varepsilon}^{\sigma}}{\partial z^{\prime}} \frac{\partial}{\partial z_{1}}\right\rangle\right.\right. \\
\left.\left.-\left\langle\overline{\zeta^{\prime}}, \frac{\partial}{\partial \bar{z}^{\prime}}-2 \sqrt{-1} \frac{\partial \varphi_{\varepsilon}^{\sigma}}{\partial \bar{z}^{\prime}} \frac{\partial}{\partial \bar{z}_{1}}\right\rangle\right) \in \mathbb{T}_{V} \tilde{V} ; \zeta^{\prime} \in \mathbb{C}^{n-1}, c>0\right\}
\end{gathered}
$$

Indeed, for any $(x, y, t) \in V, \zeta^{\prime} \in \mathbb{C}^{n-1}$ and $c>0$, choosing a positive constant $\varepsilon^{\prime}$ small enough we have

$$
\begin{aligned}
& \operatorname{Im}\left(z_{1}-\varepsilon^{\prime}\left(\sqrt{-1}\left(c+2\left\langle\zeta^{\prime}, \frac{\partial \varphi_{\varepsilon}^{\sigma}}{\partial z^{\prime}}\right\rangle\right)-\varphi_{\varepsilon}^{\sigma}\left(y^{\prime}+\varepsilon^{\prime} \operatorname{Im} \zeta^{\prime}, t\right)\right)\right) \\
= & y_{1}+\varepsilon^{\prime}\left(c+\left\langle\operatorname{Im} \zeta^{\prime}, \frac{\partial \varphi_{\varepsilon}^{\sigma}}{\partial y^{\prime}}\right\rangle\right)-\varphi_{\varepsilon}^{\sigma}\left(y^{\prime}, t\right)-\varepsilon^{\prime}\left\langle\operatorname{Im} \zeta^{\prime}, \frac{\partial \varphi_{\varepsilon}^{\sigma}}{\partial y}\right\rangle+O\left(\left(\varepsilon^{\prime}\right)^{2}\right) \\
= & \varepsilon^{\prime} c+O\left(\left(\varepsilon^{\prime}\right)^{2}\right)>0 .
\end{aligned}
$$

Thus we see that each boundary value $\mathrm{b}\left(\tilde{u}_{v}\right)(1 \leqslant v \leqslant N)$ as a hyperfunction is well-defined. Hence, denoting by SS the singularity spectrum of a hyperfunction, we obtain that $\operatorname{SS}\left(\mathrm{b}\left(\tilde{u}_{v}\right)\right) \subset Z$, where

$$
\mathbb{Z}:=\left\{\left(x, y, t ; \sqrt{-1} d z_{1}+2\left\langle\frac{\partial \varphi_{\varepsilon}^{\sigma}}{\partial z^{\prime}}, d z^{\prime}\right\rangle+\left\langle\frac{\partial \varphi_{\varepsilon}^{\sigma}}{\partial t}+\sqrt{-1} a, d t\right\rangle\right) \in T_{V}^{*} \tilde{V} ; a \in \mathbb{R}^{d}\right\} .
$$

We see that $P$ is defined on $Z$, and by (4.17) we have

$$
\left\{\begin{aligned}
\left|\operatorname{Im}\left(\frac{\eta_{i}}{\zeta_{1}}\right)\right| & =v_{i} \lambda \quad(1 \leqslant i \leqslant d), \\
\lambda & >M\left(\varphi_{\varepsilon}^{\sigma}\left(y^{\prime}, t\right)+\sum_{j=2}^{n}\left|y_{j}\right|\right)=M\left(y_{1}+\sum_{j=2}^{n}\left|y_{j}\right|\right) .
\end{aligned}\right.
$$

Moreover, by (4.18) we have 


$$
\left|\frac{\zeta_{j}}{\zeta_{1}}\right|=\left|2 \frac{\partial \varphi_{\varepsilon}^{\sigma}}{\partial z_{j}}\right|=\left|\frac{\partial \varphi_{\varepsilon}^{\sigma}}{\partial y_{j}}\right|<h_{0} \quad(2 \leqslant j \leqslant n) .
$$

and

$$
\left|\operatorname{Im}\left(\frac{\zeta_{j}}{\zeta_{1}}\right)\right|=\left|\operatorname{Im}\left(-2 \sqrt{-1} \frac{\partial \varphi_{\varepsilon}^{\sigma}}{\partial z_{j}}\right)\right|=\left|\frac{\partial \varphi_{\varepsilon}^{\sigma}}{\partial x_{j}}\right|=0 \quad(2 \leqslant j \leqslant n) .
$$

Hence by assumption [A-2], $P$ is invertible on $Z$. Further by (4.19), we have

$$
P\left(\begin{array}{c}
\mathrm{sp}_{V}\left(\mathrm{~b}\left(\tilde{u}_{1}\right)\right) \\
\vdots \\
\operatorname{sp}_{V}\left(\mathrm{~b}\left(\tilde{u}_{N}\right)\right)
\end{array}\right)=\left(\begin{array}{c}
\operatorname{sp}_{V}\left(\mathrm{~b}\left(\left(P_{\Sigma} \tilde{u}\right)_{1}\right)\right) \\
\vdots \\
\operatorname{sp}_{V}\left(\mathrm{~b}\left(\left(P_{\Sigma} \tilde{u}\right)_{N}\right)\right)
\end{array}\right)=\left(\begin{array}{c}
\operatorname{sp}_{V}\left(\mathrm{~b}\left(\tilde{f}_{1}\right)\right) \\
\vdots \\
\operatorname{sp}_{V}\left(\mathrm{~b}\left(\tilde{f}_{N}\right)\right)
\end{array}\right)=0 .
$$

Thus it follows that

$$
\left(\begin{array}{c}
\operatorname{sp}_{V}\left(\mathrm{~b}\left(\tilde{u}_{1}\right)\right) \\
\vdots \\
\operatorname{sp}_{V}\left(\mathrm{~b}\left(\tilde{u}_{N}\right)\right)
\end{array}\right)=0
$$

On the other hand, it is easy to see that for any $1 \leqslant j \leqslant n$

$$
\frac{\partial}{\partial \bar{z}_{j}}\left(\begin{array}{c}
\mathrm{b}\left(\tilde{u}_{1}\right) \\
\vdots \\
\mathrm{b}\left(\tilde{u}_{N}\right)
\end{array}\right)=0
$$

Hence the proof is complete.

Step 5. Set

$$
\begin{gathered}
W^{\sigma}:=\left\{(z, t) \in D^{\prime} ; 0 \leqslant \sigma_{j} v_{j} t_{j}(1 \leqslant j \leqslant d), 0<\sum_{j=1}^{d} \sigma_{j} v_{j} t_{j}<T_{0},\right. \\
\left.\operatorname{Im} z_{1}>\delta_{1}\left(\sum_{j=1}^{d} \sigma_{j} v_{j} t_{j}\right)^{1 /|m|}+\frac{h}{n-1} \sum_{j=2}^{n}\left|\operatorname{Im} z_{j}\right|\right\} \\
\cup\left\{(z, \tau) \in \mathbb{C}^{n+d} ; a<|\operatorname{Re} z| \leqslant a_{1},|\operatorname{Im} z|<a^{\prime},\right. \\
\left.0 \leqslant \sigma_{j} v_{j} t_{j}(1 \leqslant j \leqslant d), \sum_{j=1}^{d} \sigma_{j} v_{j} t_{j}<T_{0}\right\},
\end{gathered}
$$




$$
Z_{\varepsilon}^{\sigma}:=\widetilde{Z}_{\varepsilon}^{\sigma} \backslash W^{\sigma},
$$

where

$$
\widetilde{Z_{\varepsilon}^{\sigma}}:=\left\{(z, t) \in D^{\prime} ; 0 \leqslant \sigma_{j} v_{j} t_{j}(1 \leqslant j \leqslant d), 0<\sum_{j=1}^{d} \sigma_{j} v_{j} t_{j}<T_{0}, \quad \operatorname{Im} z_{1}=\varphi_{\varepsilon}^{\sigma}\left(\operatorname{Im} z^{\prime}, t\right)\right\} .
$$

Note that by (4.13) and (4.15) $\tilde{u}$ is analytic in some neighborhood of $W^{\sigma} \cup\left\{(z, t) \in D^{\prime} ; \prod_{j=1}^{d} t_{j}=0, \operatorname{Im} z_{1}=\varphi_{\varepsilon}^{\sigma}\left(\operatorname{Im} z^{\prime}, t\right)\right\}$. Choosing $\varepsilon$ sufficiently large, we see that $Z_{\varepsilon}^{\sigma}=\emptyset$. We will show that $Z_{\varepsilon}^{\sigma}$ is compact.

$$
\begin{aligned}
& \text { Case 1. If } 0<\sum_{j=1}^{d} \sigma_{j} v_{j} t_{j}<c_{1} \text { or } T_{0}-c_{1}<\sum_{j=1}^{d} \sigma_{j} v_{j} t_{j}<T_{0} \text {, then by (4.16) } \\
& \varphi_{\varepsilon}^{\sigma}\left(\operatorname{Im} z^{\prime}, t\right)>\varphi_{1}\left(\sum_{j=1}^{d} \sigma_{j} v_{j} t_{j}\right)+n h\left|\operatorname{Im} z^{\prime}\right|>\delta_{1}\left(\sum_{j=1}^{d} \sigma_{j} v_{j} t_{j}\right)^{1 /|m|}+h \sum_{j=2}^{n}\left|\operatorname{Im} z_{j}\right| .
\end{aligned}
$$

Hence $(z, \tau) \notin Z_{\varepsilon}^{\sigma}$.

Case 2. If $a<|\operatorname{Re} z|<a_{1}$, then $(z, \tau) \notin Z_{\varepsilon}^{\sigma}$ by definition.

Case 3. Since $M_{1} a^{\prime}>\delta_{1}$ by (4.14), we can take $a^{\prime \prime}$ as $M_{1} a^{\prime}>M_{1} a^{\prime \prime}>\delta_{1}$. Therefore, if $a^{\prime \prime}<\left|\operatorname{Im} z^{\prime}\right|<a^{\prime}$, then we have

$$
\begin{aligned}
\varphi_{\varepsilon}^{\sigma}\left(\operatorname{Im} z^{\prime}, t\right) & >M_{1}\left(\left(\sum_{j=1}^{d} \sigma_{j} v_{j} t_{j}\right)^{1 /|m|}+n h\right)\left|\operatorname{Im} z^{\prime}\right| \\
& >\delta_{1}\left(\sum_{j=1}^{d} \sigma_{j} v_{j} t_{j}\right)^{1 /|m|}+h \sum_{j=2}^{n}\left|\operatorname{Im} z_{j}\right| .
\end{aligned}
$$

Hence $(z, t) \notin Z_{\varepsilon}^{\sigma}$.

Case 4. Since $2 h<1$ and $2 \delta_{1} T_{0}{ }^{1 /|m|}<a^{\prime}$ by (4.4) and (4.14), we can choose $a^{\prime \prime \prime}$ such that $h a^{\prime}+\delta_{1} T_{0}{ }^{1 /|m|}<a^{\prime \prime \prime}<a^{\prime}$. Therefore, if $\operatorname{Im} z_{1}=\varphi_{\varepsilon}^{\sigma}\left(\operatorname{Im} z^{\prime}, t\right),|\operatorname{Re} z|<a_{1}$, $\left|\operatorname{Im} z^{\prime}\right|<a^{\prime}$ and $a^{\prime \prime \prime} \operatorname{Im} z_{1}<a^{\prime}$, then

$$
\operatorname{Im} z_{1}>a^{\prime \prime \prime}>\delta_{1}\left(\sum_{j=1}^{d} \sigma_{j} v_{j} t_{j}\right)^{1 /|m|}+\frac{h}{n-1} \sum_{j=2}^{n}\left|\operatorname{Im} z_{j}\right| .
$$


Hence $(z, t) \in W^{\sigma}$; that is, $(z, t) \notin Z_{\varepsilon}^{\sigma}$.

Thus we see that $Z_{\varepsilon}^{\sigma}$ is compact. Therefore, by the "sweeping-out" method we see that $\tilde{u}$ is analytically continued to

$$
\begin{aligned}
\left\{(z, t) \in D^{\prime} ; 0 \leqslant\right. & \sigma_{j} v_{j} t_{j}(1 \leqslant j \leqslant d), \quad 0<\sum_{j=1}^{d} \sigma_{j} v_{j} t_{j}<T_{0} \\
\operatorname{Im} z_{1}> & \exp \left(M_{1} \sum_{j=1}^{d} \sigma_{j} v_{j} t_{j}\right) \\
& \left.\times\left(\varphi_{1}\left(\sum_{j=1}^{d} \sigma_{j} v_{j} t_{j}\right)+\left(M_{1}\left(\sum_{j=1}^{d} \sigma_{j} v_{j} t_{j}\right)^{1 /|m|}+n h\right)\left|\operatorname{Im} z^{\prime}\right|\right)\right\} .
\end{aligned}
$$

For any $\varepsilon>0$ and $T^{\prime}$ with $0<T^{\prime}<T_{0}$, we can find $\varphi_{1}$ as $\varphi_{1}\left(T^{\prime}\right)<\varepsilon$. Thus, we can conclude that $\tilde{u}$ is analytic on some complex neighborhood of

$$
\begin{aligned}
U^{\sigma}:=\left\{(z, t) \in D^{\prime} ; 0 \leqslant \sigma_{j} v_{j} t_{j}(1 \leqslant j \leqslant d), \quad 0<\sum_{j=1}^{d} \sigma_{j} v_{j} t_{j}<T_{0},\right. \\
\left.\operatorname{Im} z_{1}>\exp \left(\sum_{j=1}^{d} \sigma_{j} v_{j} t_{j}\right)\left(M_{1}\left(\sum_{j=1}^{d} \sigma_{j} v_{j} t_{j}\right)^{1 /|m|}+n h\right)\left|\operatorname{Im} z^{\prime}\right|\right\} .
\end{aligned}
$$

On the other hand, there exists a sufficiently small positive constant $\varepsilon$ such that

$$
\left\{(x+\sqrt{-1}(s, 0, \ldots, 0), t) \in C^{n} \times \boldsymbol{R}^{d} ;|x|+|t|<\varepsilon, 0<s<\varepsilon\right\} \subset U_{1} \cup \bigcup_{\sigma \in\{-1,1\}^{d}} U^{\sigma}
$$

Thus, by Theorem 3.1.1 of [S-K-K] Chapter I each boundary value $\mathrm{b}\left(\tilde{u}_{v}\right)$ $(1 \leqslant v \leqslant N)$ as a hyperfunction is well-defined at $(x, t)=(0,0)$. Further, it is easy to see that $u(x, t):={ }^{t}\left(\operatorname{sp}_{M}\left(\mathrm{~b}\left(\tilde{u}_{1}\right)\right), \ldots, \mathrm{sp}_{M}\left(\mathrm{~b}\left(\tilde{u}_{N}\right)\right)\right) \in \rho_{!}\left(\left.\mathscr{C}_{M}\right|_{N_{M} \times T_{M}^{*}}\right)_{p_{0}}^{\oplus N}$. This completes the proof of Theorem 4.2.

4.5. Corollary. Let $P$ be a matrix of an analytic differential operators of Fuchsian type defined on a neighborhood of $(x, t)=(0,0)$. Assume [A-1] and the following:

[A-3]. $\operatorname{det}\left(\sigma_{\mid m^{(v) \mid}}\left(P^{(\mu, v)}\right)(z, \tau ; \zeta, \eta)\right)=\tau^{\tilde{k}} \tilde{P}(z, \tau ; \zeta, \eta)$ for a function $\tilde{P}\left(\tilde{k}:=\sum_{v=1}^{N} k^{(v)}\right.$ $\left.\in N_{0}{ }^{d}\right)$ satisfying the following:

For any $\zeta^{*} \in \boldsymbol{R} \backslash\{0\}$, there exists $\Theta \in G L(n ; \boldsymbol{R})$ such that $\Theta(1,0, \ldots, 0)=\zeta^{*}$ and that $\tilde{P}(z, t ; \Theta \zeta, \eta)$ satisfies the condition in [A-2].

Then, for any hyperfunctions with holomorphic parameters 


$$
f(x, t), g(x, t) \in\left(\left.\mathscr{B} \mathcal{O}_{L}\right|_{M}\right)_{0}^{\oplus N},
$$

there exists an $N$-tuple of hyperfunctions

$$
u(x, t) \in\left(\mathscr{B}_{M}\right)_{0}^{\oplus N}
$$

such that $u$ has $t$ as real analytic parameters and is a solution of the Goursat problem

$$
\left\{\begin{aligned}
P\left(x, t ; \partial_{x}, \partial_{t}\right) u(x, t) & =f(x, t) \\
u(x, t)-g(x, t) & =O\left(t^{m-k}\right)
\end{aligned}\right.
$$

Proof. We may assume that $g \equiv 0$. By the flabbiness of the sheaves of microfunctions and hyperfunctions and by (4.22), we can find a representation

$$
f(x, t)=\sum_{j=1}^{J} F_{j}\left((x, t)+\sqrt{-1} \Gamma_{j} 0\right),
$$

where each $N$-tuple $F_{j}=\tilde{f}_{j}+f_{j}^{\prime}$ is of type (4.8). Moreover by [A-3], we may assume that we can apply the proof of Theorem 4.2; that is, for any $1 \leqslant j \leqslant J$ we can find an $N$-tuple $\tilde{u}_{j}$ of holomorphic functions on some infinitesimal wedge-type domain $D+\sqrt{-1} \Gamma_{j}^{\prime} 0$ such that

$$
\left\{\begin{aligned}
P \tilde{u}_{j} & =\tilde{J}_{j}, \\
\tilde{u}_{j} & =O\left(t^{m-k}\right) .
\end{aligned}\right.
$$

On the other hand, since an $N$-tuple $f^{\prime}:=\sum_{j=1}^{J} f_{j}^{\prime}$ is analytic in a neighborhood of $(x, t)=(0,0)$ there exists a unique $N$-tuple $u^{\prime}$ of analytic functions such that

$$
\left\{\begin{aligned}
\mathbb{P} u^{\prime} & =f^{\prime} \\
u^{\prime} & =O\left(t^{m-k}\right)
\end{aligned}\right.
$$

by the argument to the proof of Theorem 1.3 and Madi's result [M]. Thus an $N$-tuple of hyperfunctions

$$
u(x, t)={ }^{t}\left(u_{1}(x, t), \ldots, u_{N}(x, t)\right):=\sum_{j=1}^{J} u_{j}\left((x, t)+\sqrt{-1} \Gamma_{j}^{\prime} 0\right)+u^{\prime}(x, t)
$$

is a solution and has $t$ as real analytic parameters. 


\section{References}

[Ba-G] Baouendi, M. S. and Goulaouic, C., Cauchy problems with characteristic initial hypersurface, Comm. Pure Appl. Math., 26 (1973), 455-475.

[Bo-Sc] Bony, J. M. et Schapira, P., Propagation des singularités analytiques pour les solutions des équations aux dérivées partielles, Ann. Inst. Fourier Grenoble, 26-1 (1976), 81-140.

[Bou-K] Boutet de Monvel, L. and Krée, P., Pseudo-differential operators and Gevrey classes, Ann. Inst. Fourier Grenoble, 17-1 (1967), 295-323.

[H] Hörmander, L., The Analysis of Linear Partial Differential Operators I, 2nd ed., Grundlehren Math. Wiss. 256, Springer, Berlin-Heidelberg-New York, 1990.

[K-K] Kashiwara, M. and Kawai, T., Micro-hyperbolic pseudo-differential operators I, J. Math. Soc. Japan, 27 (1975), 359-404.

$\left[\mathrm{K}^{3}\right]$ Kashiwara, M., Kawai, T. and Kimura, T., Foundation of Algebraic Analysis, Kinokuniya, Tokyo, 1980 (Japanese); English transl., Princeton Univ. Press, Princeton, 1986.

[K-Sc1] Kashiwara, M. and Schapira, P., Micro-hyperbolic systems, Acta Math., 142 (1977), $1-55$.

[K-Sc2] Sheaves on Manifolds, Grundlehren Math. Wiss. 292, Springer, BerlinHeidelberg-New York, 1990.

[Kt-To] Kataoka, K. and Tose, N., Vanishing theorems for the sheaf of microfunctions with holomorphic parameters, J. Fac. Sci. Univ. Tokyo, Sect. IA, 35 (1988), 313-320.

[La-MF] Laurent, Y. et Monterio Fernandes, T., Systèmes différentiels fuchsiens le long d'une sous-variété, Publ. RIMS, Kyoto Univ., 24 (1988), 397-431.

[M] Madi, N. S., Problème de Goursat holomorphe a variables fuchsiennes, Bull. Sci. Math., $2^{e}$ série, 111 (1987), 291-312.

[O1] Oaku, T., Micro-local cauchy problems and local boundary value problemes, Proc. Japan Acad., 55 (1979), 136-140.

[O2] The Cauchy-Kovalevskaja theorem for pseudo-differential operators of Fuchsian type and its applications, RIMS Kôkûroku, Kyoto Univ. 361, 1979, pp.131-150.

[O3] - Uniqueness of hyperfunction solutions of Fuchsian partial differential equations, RIMS Kôkûroku, Kyoto Univ. 508, 1983, pp. 41-60, (Japanese).

[O4] Microlocal boundary value problem for Fuchsian operators, I, J. Fac. Sci. Univ. Tokyo, Sect. IA, 32 (1985), 287-317.

[S-K-K] Sato, M., Kawai, T. and Kashiwara, M., Microfunctions and pseudo-differential equations, Hyperfunctions and Pseudo-Differential Equations, (Komatsu, H., ed.), Proceedings Katata 1971, Lecture Notes in Math. 287, Springer, Berlin-Heidelberg-New York, 1973, pp.265-529.

[Sz-Zi] Szmydt, Z. and Ziemian, B., The Mellin Transformation and Fuchsian Type Partial Differential Equations, Math. Appl. 56, Kluwer, Dordrecht-Boston-London, 1992.

[Ta] Tahara, H., Fuchsian type equations and Fuchsian hyperbolic equations, Japan. J. Math., 5 (1979), 245-347.

[W] Wagschal, C., Une généralisation du problème de Goursat pour des systèmes d'équations intégro-différentielles holomorphes ou partiellment holomorphes, J. Math. Pures Appl., 53 (1974), 99-132. 
